\title{
Phase transition in the Connes-Marcolli GL2-system
}

\author{
Marcelo Laca, Nadia S. Larsen, ${ }^{2}$ and Sergey Neshveyev ${ }^{3}$
}

\begin{abstract}
We develop a general framework for analyzing KMS-states on $\mathrm{C}^{*}$-algebras arising from actions of Hecke pairs. We then specialize to the system recently introduced by Connes and Marcolli and classify its KMS-states for inverse temperatures $\beta \neq 0,1$. In particular, we show that for each $\beta \in(1,2]$ there exists a unique $\mathrm{KMS}_{\beta}$-state.
\end{abstract}

Mathematics Subject Classification (2000). Primary 46L55; Secondary 58B34, 82B10, 20C08, 37A45.

Keywords. Hecke $\mathrm{C}^{*}$-algebras, KMS states, phase transition.

\section{Introduction}

More than ten years ago Bost and Connes [3] constructed a $C^{*}$-dynamical system with the Galois group $G\left(\mathbb{Q}^{\mathrm{ab}} / \mathbb{Q}\right)$ as symmetry group and with phase transition related to properties of zeta and $L$-functions. Since then there have been numerous, and only partially successful, attempts to generalize the Bost-Connes system to arbitrary number fields, see [5, Section 1.4] for a survey. As was later emphasized by Connes, the BC-system has yet another remarkable property: there exists a dense $\mathbb{Q}$-subalgebra such that the maximal abelian extension $\mathbb{Q}^{\mathrm{ab}}$ of $\mathbb{Q}$ arises as the set of values of a ground state of the system on it. If one puts this property as a requirement for an arbitrary number field, one recognizes that the problem of finding the right analogue of the BC-system is related to Hilbert's 12th problem on explicit class field theory. Since the only case (in addition to $\mathbb{Q}$ ) for which Hilbert's problem is completely solved is that of imaginary quadratic fields, these fields should be the first to investigate. This has been done in recent papers of Connes, Marcolli and Ramachandran [5], [6], [7], [8]. Connes and Marcolli [5], [6] constructed a $\mathrm{GL}_{2}$-system, an analogue of the BC-system with $\mathbb{Q}^{*}$ replaced by $\mathrm{GL}_{2}(\mathbb{Q})$. Its specialization to a subsystem

\footnotetext{
${ }^{1}$ Part of this work was carried out through several visits of the author to the Department of Mathematics at the University of Oslo. He would like to thank the department for their hospitality, and the SUPREMA project for the support. Supported by the Natural Sciences and Engineering Research Council of Canada.

${ }^{2}$ Supported by the Research Council of Norway.

${ }^{3}$ Supported by the Research Council of Norway.
} 
compatible with complex multiplication in a given imaginary quadratic field gives the right analogue of the BC-system for such a field [7], [8]. Later Ha and Paugam [12], inspired by constructions of Connes and Marcolli, proposed an analogue of the BCsystem for an arbitrary number field.

Connes and Marcolli classified KMS-states of the $\mathrm{GL}_{2}$-system for inverse temperatures $\beta \notin(1,2]$. It is the primary goal of the present paper to elucidate what happens in the critical region $(1,2]$. Along the way we develop some general tools for analyzing systems of the type introduced by Connes and Marcolli, which can be thought of as crossed products of abelian algebras by Hecke algebras.

Our approach to the problem is along the lines of that of the first author in the case of the BC-system [15]. Namely, in Proposition 3.2 we show that KMS-states correspond to states on the diagonal subalgebra which are scaled by the action of $\mathrm{GL}_{2}^{+}(\mathbb{Q})$, or rather by the Hecke operators. As our first application we recover in Theorem 3.7 the results of Connes and Marcolli. We then prove our main result, Theorem 4.1, the uniqueness of a $\mathrm{KMS}_{\beta}$-state for each $\beta \in(1,2]$. The strategy is similar to that of the third author in the BC-case [18]. Namely, we prove the uniqueness and ergodicity, under the action of $\mathrm{GL}_{2}^{+}(\mathbb{Q})$, of the measure defining a symmetric $\mathrm{KMS}_{\beta}$-state by analyzing an explicit formula for the projection onto the space of $\mathrm{Mat}_{2}^{+}(\mathbb{Z})$-invariant functions, see Lemma 4.4 and Corollary 4.7, and then derive from this the main uniqueness result. There are two main complications compared to the $\mathrm{BC}$-case. The first is that instead of semigroup actions we now have to deal with representations of Hecke algebras. The second is the presence in the system of a continuous component corresponding to the infinite place. As a result, the critical step now is to prove the uniqueness of a symmetric, that is, $\mathrm{GL}_{2}(\hat{\mathbb{Z}})$-invariant, $\mathrm{KMS}_{\beta}$-state, while in the $\mathrm{BC}$ case the analogous statement is almost obvious. To show this uniqueness we use a deep result of Clozel, Oh and Ullmo [4] on equidistribution of Hecke points. We point out that, as opposed to the $\mathrm{BC}$-case, there are many symmetric states for $\beta>2$, which can be easily seen from Theorem 3.7 below.

\section{Proper actions and groupoid $\mathrm{C}^{*}$-algebras}

Let $G$ be a countable group acting on a locally compact second countable space $X$. The reduced crossed product $C_{0}(X) \rtimes_{\mathrm{r}} G$ is the reduced $\mathrm{C}^{*}$-algebra of the transformation groupoid $G \times X$ with unit space $X$, source and range maps $(g, x) \mapsto x$ and $(g, x) \mapsto$ $g x$, respectively, and the product

$$
(g, h x)(h, x)=(g h, x) .
$$

If the restriction of the action to a subgroup $\Gamma$ of $G$ is free and proper, we can introduce a new groupoid $\Gamma \backslash G \times_{\Gamma} X$ by taking the quotient of $G \times X$ by the action of $\Gamma \times \Gamma$ defined by

$$
\left(\gamma_{1}, \gamma_{2}\right)(g, x)=\left(\gamma_{1} g \gamma_{2}^{-1}, \gamma_{2} x\right)
$$


Thus the unit space of $\Gamma \backslash G \times_{\Gamma} X$ is $\Gamma \backslash X$, and the product is induced from that on $G \times X$. This groupoid is Morita equivalent in the sense of [17] to the transformation groupoid $G \times X$. Although we will not need this result, let us briefly recall the argument. By definition of Morita equivalence first of all we have to find a space $Z$ with commuting actions of our groupoids. We take $Z=G \times_{\Gamma} X$, the quotient of $G \times X$ by the action of $\Gamma$ given by $\gamma(g, x)=\left(g \gamma^{-1}, \gamma x\right)$. The left and right actions of the groupoid $G \times X$ on itself induce a left action of $G \times X$ and a right action of $\Gamma \backslash G \times_{\Gamma} X$ on $Z$. The map $Z \rightarrow \Gamma \backslash X, \Gamma(g, x) \mapsto \Gamma x$, induces a homeomorphism between the quotient of $Z$ by the action of $G \times X$ and the unit space $\Gamma \backslash X$ of the groupoid $\Gamma \backslash G \times_{\Gamma} X$. Similarly, the map $Z \rightarrow X, \Gamma(g, x) \mapsto g x$, induces a homeomorphism between the quotient of $Z$ by $\Gamma \backslash G \times_{\Gamma} X$ and $X$. Thus the groupoids are indeed Morita equivariant. Recall then that by [17], Theorem 2.8, the corresponding reduced $\mathrm{C}^{*}$-algebras are Morita equivalent.

If the action of $\Gamma$ is proper but not free, the quotient space $\Gamma \backslash G \times_{\Gamma} X$ is no longer a groupoid, since the composition of classes using representatives will in general depend on the choice of representatives. As was observed in [9] and [5], nevertheless, the same formula for convolution of two functions as in the groupoid case gives us a well-defined algebra, and by completion we get a $\mathrm{C}^{*}$-algebra. In more detail, consider the space $C_{\mathrm{c}}\left(\Gamma \backslash G \times_{\Gamma} X\right)$ of continuous compactly supported functions on $\Gamma \backslash G \times_{\Gamma} X$. We consider its elements as $(\Gamma \times \Gamma)$-invariant functions on $G \times X$, and define a convolution of two such functions by

$$
\left(f_{1} * f_{2}\right)(g, x)=\sum_{h \in \Gamma \backslash G} f_{1}\left(g h^{-1}, h x\right) f_{2}(h, x) .
$$

To see that the convolution is well defined, assume the support of $f_{i}$ is contained in $(\Gamma \times \Gamma)\left(\left\{g_{i}\right\} \times U_{i}\right)$, where $g_{i} \in G$ and $U_{i}$ is a compact subset of $X$. Let $\left\{\gamma_{1}, \ldots, \gamma_{n}\right\}$ be the set of all elements $\gamma \in \Gamma$ such that $\gamma g_{2} U_{2} \cap U_{1} \neq \emptyset$. Note that this set is finite since the action of $\Gamma$ is assumed to be proper. If $f_{2}(h, x) \neq 0$ then there exists $\gamma \in \Gamma$ such that $h \gamma^{-1} \in \Gamma g_{2}$ and $\gamma x \in U_{2}$. Since the number of $\gamma$ 's such that $\gamma x \in U_{2}$ is finite, we already see that the sum above is finite. If furthermore $f_{1}\left(g h^{-1}, h x\right) \neq 0$ then replacing $h$ by another representative of the right coset $\Gamma h$ we may assume that $g h^{-1} \in \Gamma g_{1}$ and $h x \in U_{1}$. Then if $h \gamma^{-1}=\tilde{\gamma} g_{2}$ with $\tilde{\gamma} \in \Gamma$, we get $h x=\tilde{\gamma} g_{2} \gamma x \in$ $\tilde{\gamma} g_{2} U_{2}$. Hence $\tilde{\gamma}=\gamma_{i}$ for some $i$, and therefore $g \in \Gamma g_{1} h=\Gamma g_{1} \gamma_{i} g_{2} \gamma$. Thus the support of $f_{1} * f_{2}$ is contained in the union of the sets $(\Gamma \times \Gamma)\left(\left\{g_{1} \gamma_{i} g_{2}\right\} \times U_{2}\right)$, so $f_{1} * f_{2} \in C_{\mathrm{c}}\left(\Gamma \backslash G \times_{\Gamma} X\right)$ and the latter space becomes an algebra. It is not difficult to check that the convolution is associative.

Define also an involution on $C_{\mathrm{c}}\left(\Gamma \backslash G \times_{\Gamma} X\right)$ by

$$
f^{*}(g, x)=\overline{f\left((g, x)^{-1}\right)}=\overline{f\left(g^{-1}, g x\right)} .
$$

If the support of $f$ is contained in $(\Gamma \times \Gamma)\left(\left\{g_{0}\right\} \times U\right)$ for $g_{0} \in G$ and compact 
$U \subset X$, then the support of $f^{*}$ is contained in

$$
\left((\Gamma \times \Gamma)\left(\left\{g_{0}\right\} \times U\right)\right)^{-1}=(\Gamma \times \Gamma)\left(\left\{g_{0}\right\} \times U\right)^{-1}=(\Gamma \times \Gamma)\left(\left\{g_{0}^{-1}\right\} \times g_{0} U\right),
$$

so indeed $f^{*} \in C_{\mathrm{c}}\left(\Gamma \backslash G \times_{\Gamma} X\right)$.

For each $x \in X$ we define a $*$-representation $\pi_{x}: C_{\mathrm{c}}\left(\Gamma \backslash G \times_{\Gamma} X\right) \rightarrow B\left(\ell^{2}(\Gamma \backslash G)\right)$ by

$$
\pi_{x}(f) \delta_{\Gamma h}=\sum_{g \in \Gamma \backslash G} f\left(g h^{-1}, h x\right) \delta_{\Gamma g},
$$

where $\delta_{\Gamma g}$ denotes the characteristic function of the coset $\Gamma g$. It is standard to show that the operators $\pi_{x}(f)$ are bounded, but we include a proof for the reader's convenience.

Lemma 1.1. For each $f \in C_{\mathrm{c}}\left(\Gamma \backslash G \times_{\Gamma} X\right)$ the operators $\pi_{x}(f), x \in X$, are uniformly bounded.

Proof. For $\xi_{1}, \xi_{2} \in \ell^{2}(\Gamma \backslash G)$ we have

$$
\begin{aligned}
& \left|\left(\pi_{x}(f) \xi_{1}, \xi_{2}\right)\right| \\
& \quad \leq \sum_{g, h \in \Gamma \backslash G}\left|f\left(g h^{-1}, h x\right)\right|\left|\xi_{1}(h)\right|\left|\xi_{2}(g)\right| \\
& \quad \leq\left(\sum_{g, h \in \Gamma \backslash G}\left|f\left(g h^{-1}, h x\right)\right|\left|\xi_{1}(h)\right|^{2}\right)^{1 / 2}\left(\sum_{g, h \in \Gamma \backslash G}\left|f\left(g h^{-1}, h x\right)\right|\left|\xi_{2}(g)\right|^{2}\right)^{1 / 2} .
\end{aligned}
$$

Thus if we denote by $\|f\|_{I}$ the quantity

$$
\max \left\{\sup _{x \in X, h \in G} \sum_{g \in \Gamma \backslash G}\left|f\left(g h^{-1}, h x\right)\right|, \sup _{x \in X, g \in G} \sum_{h \in \Gamma \backslash G}\left|f\left(g h^{-1}, h x\right)\right|\right\},
$$

we get $\left\|\pi_{x}(f)\right\| \leq\|f\|_{I}$ for any $x \in X$, so it suffices to show that $\|f\|_{I}$ is finite. Replacing $x$ by $h^{-1} x$ and $g$ by $g h$ in the first supremum above, we see that this supremum equals

$$
\|f\|_{I, s}:=\sup _{x \in X} \sum_{g \in \Gamma \backslash G}|f(g, x)| .
$$

Observe next that $f\left(g h^{-1}, h x\right)=\overline{f^{*}\left(h g^{-1}, g x\right)}$, so that the second supremum is equal to $\left\|f^{*}\right\|_{I, s}$. Therefore $\|f\|_{I}=\max \left\{\|f\|_{I, s},\left\|f^{*}\right\|_{I, s}\right\}$. It remains to show that $\|f\|_{I, s}$ is finite for any $f \in C_{\mathrm{c}}\left(\Gamma \backslash G \times_{\Gamma} X\right)$.

Assume the support of $f$ is contained in $(\Gamma \times \Gamma)\left(\left\{g_{0}\right\} \times U\right)$ for some $g_{0} \in G$ and compact $U \subset X$. Since the action of $\Gamma$ is proper, there exists $n \in \mathbb{N}$ such that the sets $\gamma_{i} U, i=1, \ldots, n+1$, have trivial intersection for any different $\gamma_{1}, \ldots, \gamma_{n+1} \in \Gamma$. Now if $f(g, x) \neq 0$ for some $g$ and $x$, there exists $\gamma \in \Gamma$ such that $g \gamma^{-1} \in \Gamma g_{0}$ and 
$\gamma x \in U$. Since the number of $\gamma$ 's such that $\gamma x \in U$ is at most $n$, we see that for each $x \in X$ the sum in the definition of $\|f\|_{I, s}$ has at most $n$ nonzero summands. Hence $\|f\|_{I, S}$ is finite, and the proof of the lemma is complete.

We denote by $C_{\mathrm{r}}^{*}\left(\Gamma \backslash G \times_{\Gamma} X\right)$ the completion of $C_{\mathrm{c}}\left(\Gamma \backslash G \times_{\Gamma} X\right)$ in the norm defined by the representation $\bigoplus_{x \in X} \pi_{x}$, that is,

$$
\|f\|=\sup _{x \in X}\left\|\pi_{x}(f)\right\| \text {. }
$$

Denoting by $U_{g}$ the unitary operator on $\ell^{2}(\Gamma \backslash G)$ such that $U_{g} \delta_{\Gamma h}=\delta_{\Gamma h g-1}$, we get $U_{g} \pi_{x}(f) U_{g}^{*}=\pi_{g x}(f)$. Hence $\left\|\pi_{x}(f)\right\|=\left\|\pi_{g x}(f)\right\|$ and so the supremum above is actually over $G \backslash X$.

Using the embedding $X \hookrightarrow G \times X, x \mapsto(e, x)$, we may consider $\Gamma \backslash X$ as an open subset of $\Gamma \backslash G \times_{\Gamma} X$, and then the algebra $C_{0}(\Gamma \backslash X)$ as a subalgebra of $C_{\mathrm{r}}^{*}\left(\Gamma \backslash G \times_{\Gamma} X\right)$. More generally, any bounded continuous function on $\Gamma \backslash X$ defines a multiplier of $C_{\mathrm{r}}^{*}\left(\Gamma \backslash G \times_{\Gamma} X\right)$.

Lemma 1.2. There exists a conditional expectation $E: C_{\mathrm{r}}^{*}\left(\Gamma \backslash G \times_{\Gamma} X\right) \rightarrow C_{0}(\Gamma \backslash X)$ such that

$$
E(f)(x)=f(e, x) \text { for } f \in C_{\mathrm{c}}\left(\Gamma \backslash G \times_{\Gamma} X\right) .
$$

Proof. For each $x \in X$ define a state $\omega_{x}$ on $C_{\mathrm{r}}^{*}\left(\Gamma \backslash G \times_{\Gamma} X\right)$ by

$$
\omega_{x}(a)=\left(\pi_{x}(a) \delta_{\Gamma}, \delta_{\Gamma}\right) .
$$

Then the function $E(a)$ on $X$ defined by $E(a)(x)=\omega_{x}(a)$ is bounded by $\|a\|$. Since $E(f)(x)=f(e, x)$ for $f \in C_{\mathrm{c}}\left(\Gamma \backslash G \times_{\Gamma} X\right)$, we conclude that $E(a) \in C_{0}(\Gamma \backslash X)$ for every $a \in C_{\mathrm{r}}^{*}\left(\Gamma \backslash G \times_{\Gamma} X\right)$. Thus $E$ is the required conditional expectation.

Let $Y \subset X$ be a $\Gamma$-invariant clopen subset. Then, as we already observed, the characteristic function $\mathbb{1}_{\Gamma \backslash Y}$ of the set $\Gamma \backslash Y$ is an element of the multiplier algebra of $C_{\mathrm{r}}^{*}\left(\Gamma \backslash G \times_{\Gamma} X\right)$. Denote by $\Gamma \backslash G \otimes_{\Gamma} Y$ the quotient of the space

$$
\{(g, x) \mid g \in G, x \in Y, g x \in Y\}
$$

by the action of $\Gamma \times \Gamma$ defined as in (1.1). Then

$$
\mathbb{1}_{\Gamma \backslash Y} C_{\mathrm{c}}\left(\Gamma \backslash G \times_{\Gamma} X\right) \mathbb{1}_{\Gamma \backslash Y}=C_{\mathrm{c}}\left(\Gamma \backslash G \otimes_{\Gamma} Y\right) .
$$

Thus the algebra $\mathbb{1}_{\Gamma \backslash Y} C_{\mathrm{r}}^{*}\left(\Gamma \backslash G \times_{\Gamma} X\right) \mathbb{1}_{\Gamma \backslash Y}$, which we shall denote by $C_{\mathrm{r}}^{*}\left(\Gamma \backslash G \bigotimes_{\Gamma} Y\right)$, is a completion of the algebra of compactly supported functions on $\Gamma \backslash G \bigotimes_{\Gamma} Y$ with convolution product given by

$$
\left(f_{1} * f_{2}\right)(g, y)=\sum_{h \in \Gamma \backslash G: h y \in Y} f_{1}\left(g h^{-1}, h y\right) f_{2}(h, y),
$$


and involution

$$
f^{*}(g, y)=\overline{f\left(g^{-1}, g y\right)} .
$$

Note that $\pi_{x}\left(\mathbb{1}_{\Gamma \backslash Y}\right)$ is the projection onto the subspace $\ell^{2}\left(\Gamma \backslash G_{x}\right)$ of $\ell^{2}(\Gamma \backslash G)$, where the subset $G_{x}$ of $G$ is defined by

$$
G_{x}=\{g \in G \mid g x \in Y\},
$$

and then

$$
\pi_{x}(f) \delta_{\Gamma h}=\sum_{g \in \Gamma \backslash G_{x}} f\left(g h^{-1}, h x\right) \delta_{\Gamma g}
$$

for $h \in G_{x}$ and $f \in C_{\mathrm{c}}\left(\Gamma \backslash G \otimes_{\Gamma} Y\right)$. In particular, $\pi_{x}(f)=0$ if $x \notin G Y$. As we already remarked, the representations $\pi_{x}$ and $\pi_{g x}$ are unitarily equivalent for any $g \in G$. Thus we may conclude that $C_{\mathrm{r}}^{*}\left(\Gamma \backslash G \otimes_{\Gamma} Y\right)$ is precisely the completion of $C_{\mathrm{c}}\left(\Gamma \backslash G \otimes_{\Gamma} Y\right)$ in the norm

$$
\|f\|=\sup _{y \in Y}\left\|\pi_{y}(f)\right\| .
$$

This is how the algebra $C_{\mathrm{r}}^{*}\left(\Gamma \backslash G \otimes_{\Gamma} Y\right)$ was defined (in a particular case) in [5], Proposition 1.23.

Returning to the algebra $C_{\mathrm{r}}^{*}\left(\Gamma \backslash G \times_{\Gamma} X\right)$, our next goal is to show that under an extra assumption its multiplier algebra contains other interesting elements in addition to the $\Gamma$-invariant functions on $X$.

Recall that $(G, \Gamma)$ is called a Hecke pair if $\Gamma$ and $g \Gamma g^{-1}$ are commensurable for any $g \in G$, that is, $\Gamma \cap g \Gamma g^{-1}$ is a subgroup of $\Gamma$ of finite index. Equivalently, every double coset of $\Gamma$ contains finitely many right (and left) cosets of $\Gamma$, so that

$$
R_{\Gamma}(g):=|\Gamma \backslash \Gamma g \Gamma|<\infty \quad \text { for any } g \in G .
$$

Then the space $\mathscr{H}(G, \Gamma)$ of finitely supported functions on $\Gamma \backslash G / \Gamma$ is a $*$-algebra with product

$$
\left(f_{1} * f_{2}\right)(g)=\sum_{h \in \Gamma \backslash G} f_{1}\left(g h^{-1}\right) f_{2}(h)
$$

and involution $f^{*}(g)=\overline{f\left(g^{-1}\right)}$, see e.g. [13]. This algebra is represented on $\ell^{2}(\Gamma \backslash G)$ by

$$
f \delta_{\Gamma h}=\sum_{g \in \Gamma \backslash G} f\left(g h^{-1}\right) \delta_{\Gamma g},
$$

see [3]. The corresponding completion is called the reduced Hecke $\mathrm{C}^{*}$-algebra of $(G, \Gamma)$ and denoted by $C_{\mathrm{r}}^{*}(G, \Gamma)$. We shall denote by $[g]$ the characteristic function of the double coset $\Gamma g \Gamma$ considered as an element of the Hecke algebra. 
We may consider elements of $\mathscr{H}(G, \Gamma)$ as continuous functions on $\Gamma \backslash G \times_{\Gamma} X$. Although these functions are not compactly supported in general, the formulas defining the $*$-algebra structure and the regular representation of $\mathscr{H}(G, \Gamma)$ coincide with (1.2)-(1.4). Furthermore, the convolution of an element of $\mathscr{H}(G, \Gamma)$ with a compactly supported function on $\Gamma \backslash G \times_{\Gamma} X$ gives a compactly supported function. Indeed, if $f_{1}=\left[g_{1}\right]$ and the support of $f_{2} \in C_{\mathrm{c}}\left(\Gamma \backslash G \times_{\Gamma} X\right)$ is contained in $(\Gamma \times \Gamma)\left(\left\{g_{2}\right\} \times U\right)$ for a compact $U \subset X$, then the support of $f_{1} * f_{2}$ is contained in $(\Gamma \times \Gamma)\left(g_{1} \Gamma g_{2} \times U\right)$. Since $\Gamma \backslash \Gamma g_{1} \Gamma g_{2}$ is finite, we see that $f_{1} * f_{2}$ is compactly supported on $\Gamma \backslash G \times \Gamma X$. We may therefore conclude the following.

Lemma 1.3. If $(G, \Gamma)$ is a Hecke pair, then the reduced Hecke $C^{*}$-algebra $C_{\mathrm{r}}^{*}(G, \Gamma)$ is contained in the multiplier algebra of the $C^{*}$-algebra $C_{\mathrm{r}}^{*}\left(\Gamma \backslash G \times_{\Gamma} X\right)$.

It is then tempting to think of $C_{\mathrm{r}}^{*}\left(\Gamma \backslash G \times_{\Gamma} X\right)$ as a crossed product of $C_{0}(\Gamma \backslash X)$ by an action of the Hecke pair $(G, \Gamma)$. This point of view has been formalized by Tzanev [21] who introduced a notion of a crossed product of an algebra by an action of a Hecke pair.

Remark 1.4. We defined $C_{\mathrm{r}}^{*}\left(\Gamma \backslash G \times_{\Gamma} X\right)$ assuming that the action of $\Gamma$ on $X$ is proper. It is however easy to see that the construction makes sense under the following weaker assumptions: $\Gamma \backslash G \times_{\Gamma} X$ is Hausdorff, and if for a compact set $K \subset X$ we put $\Gamma_{K}=\{\gamma \in \Gamma \mid \gamma K \cap K \neq \emptyset\}$ then the set $\Gamma \backslash \Gamma g \Gamma_{K}$ is finite for any $g \in G$. Note that the second assumption is automatically satisfied when $(G, \Gamma)$ is a Hecke pair.

\section{Dynamics and KMS-states}

Assume as above that we have an action of $G$ on $X$ such that the action of $\Gamma \subset G$ is proper, and $Y \subset X$ is a $\Gamma$-invariant clopen set. Assume now that we are given a homomorphism

$$
N: G \rightarrow \mathbb{R}_{+}^{*}=(0,+\infty)
$$

such that $\Gamma$ is contained in the kernel of $N$. Then we define a one-parameter group of automorphisms of $C_{\mathrm{r}}^{*}\left(\Gamma \backslash G \times_{\Gamma} X\right)$ by

$$
\sigma_{t}(f)(g, x)=N(g)^{i t} f(g, x) \text { for } f \in C_{\mathrm{c}}\left(\Gamma \backslash G \times_{\Gamma} X\right) .
$$

More precisely, if we denote by $\bar{N}$ the selfadjoint operator on $\ell^{2}(\Gamma \backslash G)$ defined by

$$
\bar{N} \delta_{\Gamma g}=N(g) \delta_{\Gamma g},
$$

then the dynamics $\sigma_{t}$ is spatially implemented by the unitary operator $\bigoplus_{x \in X} \bar{N}^{i t}$ on $\bigoplus_{x \in X} \ell^{2}(\Gamma \backslash G)$. In other words,

$$
\pi_{x}\left(\sigma_{t}(a)\right)=\bar{N}^{i t} \pi_{x}(a) \bar{N}^{-i t} \text { for all } x \in X .
$$


Recall, see e.g. [14], that a semifinite $\sigma$-invariant weight $\varphi$ is called a $\sigma-\mathrm{KMS}_{\beta^{-}}$ weight if

$$
\varphi\left(a a^{*}\right)=\varphi\left(\sigma_{i \beta / 2}(a)^{*} \sigma_{i \beta / 2}(a)\right)
$$

for any $\sigma$-analytic element $a$. The following result will be the basis of our analysis of KMS-weights.

Proposition 2.1. Assume the action of $G$ on $X$ is free, so that in particular $\Gamma \backslash G \bigotimes_{\Gamma} Y$ is a genuine groupoid. Then for any $\beta \in \mathbb{R}$ there exists a one-to-one correspondence between $\sigma-K M S_{\beta}$ weights $\varphi$ on $C_{\mathrm{r}}^{*}\left(\Gamma \backslash G \nabla_{\Gamma} Y\right)$ with domain of definition containing $C_{\mathrm{c}}(\Gamma \backslash Y)$ and Radon measures $\mu$ on $Y$ such that

$$
\mu(g Z)=N(g)^{-\beta} \mu(Z)
$$

for every $g \in G$ and every compact subset $Z \subset Y$ such that $g Z \subset Y$. Namely, such a measure $\mu$ is $\Gamma$-invariant, so it determines a measure $v$ on $\Gamma \backslash Y$ such that

$$
\int_{Y} f(y) d \mu(y)=\int_{\Gamma \backslash Y}\left(\sum_{y \in p^{-1}(\{t\})} f(y)\right) d \nu(t) \quad \text { for } f \in C_{\mathrm{c}}(Y),
$$

where $p: Y \rightarrow \Gamma \backslash Y$ is the quotient map, and the associated weight $\varphi$ is given by

$$
\varphi(a)=\int_{\Gamma \backslash Y} E(a)(x) d v(x),
$$

where $E$ is the conditional expectation defined in Lemma 1.2.

Proof. For $\Gamma=\{e\}$ the result is well-known, see e.g. [19], Proposition II.5.4. For arbitrary $\Gamma$ the result can be deduced from the fact that the $\mathrm{C}^{*}$-algebra $C_{\mathrm{r}}^{*}\left(\Gamma \backslash G \otimes_{\Gamma} Y\right)$ is Morita equivalent to the $\mathrm{C}^{*}$-algebra $\mathbb{1}_{Y}\left(C_{0}(X) \rtimes_{\mathrm{r}} G\right) \mathbb{1}_{Y}$ and general results on KMS-weights on Morita equivalent algebras, see [16], Theorem 3.2. However, a more elementary way is to argue as follows.

Since the action of $\Gamma$ on $Y$ is free, the quotient space $\Gamma \backslash G \otimes_{\Gamma} Y$ is an etale groupoid. In fact it is an etale equivalence relation on $\Gamma \backslash Y$, or an $r$-discrete principal groupoid in the terminology of [19]. To see this we have to check that the isotropy group of every point in $\Gamma \backslash Y$ is trivial, that is, if $g \in G$ is such that $g y \in Y$ and $p(g y)=p(y)$ for some $y \in Y$ then $(g, y)$ belongs to the $(\Gamma \times \Gamma)$-orbit of $(e, y)$. But if $p(g y)=p(y)$, there exists $\gamma \in \Gamma$ such that $\gamma g y=y$. Then $\gamma g=e$, since the action of $G$ is free, and therefore $(g, y)=\left(\gamma^{-1}, e\right)(e, y)$.

It is then standard to show using [19], Proposition II.5.4, that $\sigma-\mathrm{KMS}_{\beta}$ weights (with domain of definition containing $C_{\mathrm{c}}(\Gamma \backslash Y)$ ) on the $\mathrm{C}^{*}$-algebra $C_{\mathrm{r}}^{*}\left(\Gamma \backslash G \otimes_{\Gamma} Y\right)$ of the etale equivalence relation are in one-to-one correspondence with measures $v$ on $\Gamma \backslash Y$ with Radon-Nikodym cocycle $(p(y), p(g y)) \mapsto N(g)^{\beta}$. The latter means the following, see [19], Definition I.3.4. Assume $Y_{0}$ is an open subset of $Y$ such that 
the map $p: Y \rightarrow \Gamma \backslash Y$ is injective on $Y_{0}$, and $g \in G$ is such that $g Y_{0} \subset Y$. Define an injective map $\tilde{g}: p\left(Y_{0}\right) \rightarrow p\left(g Y_{0}\right)$ by $\tilde{g} p(y)=p(g y)$ for $y \in Y_{0}$, and let $\tilde{g}_{*} \nu$ be the push-forward of the measure $v$ under the map $\tilde{g}$, that is, $\tilde{g}_{*} v(Z)=v\left(\tilde{g}^{-1}(Z)\right)$ for $Z \subset p\left(g Y_{0}\right)$. Then

$$
\frac{d \tilde{g}_{*} \nu}{d \nu}=N(g)^{\beta} \quad \text { on } p\left(g Y_{0}\right) .
$$

If we denote by $\mu$ the $\Gamma$-invariant measure on $Y$ corresponding to $v$ via (2.2), then to say that the Radon-Nikodym cocycle of $v$ is $(p(y), p(g y)) \mapsto N(g)^{\beta}$ is the same as saying that $\mu$ satisfies the scaling condition (2.1).

It will be convenient to extend the measure $\mu$ to the set $G Y$.

Lemma 2.2. If $\mu$ is a measure on $Y$ as in Proposition 2.1, then it extends uniquely to a Radon measure on $G Y \subset X$ satisfying (2.1) for $Z \subset G Y$ and $g \in G$.

Proof. A more general result on extensions of KMS-weights is proved in [16], but the present particular case has the following elementary proof. Choose Borel subsets $Y_{i} \subset Y$ and elements $g_{i} \in G$ such that $G Y$ is the disjoint union of the sets $g_{i}^{-1} Y_{i}$. There is only one choice for a measure extending $\mu$ and satisfying (2.1) on $G Y$, namely, for a Borel subset $Z \subset G Y$ let

$$
\mu(Z)=\sum_{i} N\left(g_{i}\right)^{\beta} \mu\left(g_{i} Z \cap Y_{i}\right) .
$$

To show that $\mu(Z)$ is independent of any choices and that the extension satisfies (2.1), assume $G Y$ is a disjoint union of sets $h_{j}^{-1} Z_{j}$ for some $h_{j} \in G$ and Borel $Z_{j} \subset Y$. Let $g \in G$. Then

$$
\begin{aligned}
\sum_{i} N\left(g_{i}\right)^{\beta} & \mu\left(g_{i} g Z \cap Y_{i}\right) \\
& =\sum_{i} N\left(g_{i}\right)^{\beta} \sum_{j} \mu\left(g_{i} g Z \cap Y_{i} \cap g_{i} g h_{j}^{-1} Z_{j}\right) \\
& =\sum_{i} N\left(g_{i}\right)^{\beta} \sum_{j} N\left(g_{i} g h_{j}^{-1}\right)^{-\beta} \mu\left(h_{j} Z \cap h_{j} g^{-1} g_{i}^{-1} Y_{i} \cap Z_{j}\right) \\
& =N(g)^{-\beta} \sum_{j} N\left(h_{j}\right)^{\beta} \sum_{i} \mu\left(h_{j} Z \cap h_{j} g^{-1} g_{i}^{-1} Y_{i} \cap Z_{j}\right) \\
& =N(g)^{-\beta} \sum_{j} N\left(h_{j}\right)^{\beta} \mu\left(h_{j} Z \cap Z_{j}\right) .
\end{aligned}
$$

Taking $g=e$ we see that the extension of $\mu$ to $G Y$ is well defined. But then for arbitrary $g$ the above identity reads as $\mu(g Z)=N(g)^{-\beta} \mu(Z)$. 
Remark 2.3. In the notation of Proposition 2.1 choose a $\mu$-measurable subset $U$ of $Y$ such that $p: Y \rightarrow \Gamma \backslash Y$ is injective on $U$ and $p(U)=\Gamma \backslash Y$. Then the map $p$ induces an isomorphism between the restriction $\mathcal{R}_{G, U}$ of the $G$-orbit equivalence relation on $X$ to $U$ and the principal groupoid $\Gamma \backslash G \bigotimes_{\Gamma} Y$. Hence $\pi_{\varphi}\left(C_{\mathrm{r}}^{*}\left(\Gamma \backslash G \bigotimes_{\Gamma} Y\right)\right)^{\prime \prime}$ is isomorphic to the von Neumann algebra $W^{*}\left(\mathcal{R}_{G, U}, \mu\right)$ of $\left(\mathcal{R}_{G, U}, \mu\right)$, see [11]. Extend the measure $\mu$ to a $G$-quasi-invariant measure on $G Y$, which we still denote by $\mu$. Then $W^{*}\left(\mathcal{R}_{G, U}, \mu\right)$ is the reduction of the von Neumann algebra of the $G$-orbit equivalence relation on $(G Y, \mu)$ by the projection $\mathbb{1}_{U}$. Therefore

$$
\pi_{\varphi}\left(C_{\mathrm{r}}^{*}\left(\Gamma \backslash G \otimes_{\Gamma} Y\right)\right)^{\prime \prime} \cong \mathbb{1}_{U}\left(L^{\infty}(G Y, \mu) \rtimes G\right) \mathbb{1}_{U} .
$$

In some cases an argument similar to the proof of Lemma 2.2 allows us to describe all measures satisfying (2.1).

Lemma 2.4. Let $Y_{0}$ be a $\Gamma$-invariant Borel subset of $Y$ such that

(i) if $g Y_{0} \cap Y_{0} \neq \emptyset$ for some $g \in G$ then $g \in \Gamma$;

(ii) for any $y \in Y$ there exists $g \in G$ such that $g y \in Y_{0}$.

Then any $\Gamma$-invariant Borel measure on $Y_{0}$ extends uniquely to a Borel measure on $Y$ satisfying (2.1).

Proof. Let $\mu_{0}$ be a $\Gamma$-invariant measure on $Y_{0}$. Since the assumptions imply that $Y$ is a disjoint union of translates of $Y_{0}$ by representatives of the right cosets of $\Gamma$, that is, $Y=\bigsqcup_{h \in \Gamma \backslash G}\left(h^{-1} Y_{0} \cap Y\right)$, there is only one choice for a measure $\mu$ extending $\mu_{0}$ and satisfying (2.1), namely,

$$
\mu(Z)=\sum_{h \in \Gamma \backslash G} N(h)^{\beta} \mu_{0}\left(h Z \cap Y_{0}\right) .
$$

Since $\mu_{0}$ is $\Gamma$-invariant, $\mu(Z)$ is independent of the choice of representatives, so all we need to check is that (2.1) holds. Let $g \in G$. Then

$$
\begin{aligned}
\mu(g Z) & =\sum_{h \in \Gamma \backslash G} N(h)^{\beta} \mu_{0}\left(h g Z \cap Y_{0}\right) \\
& =N(g)^{-\beta} \sum_{h \in \Gamma \backslash G} N(h g)^{\beta} \mu_{0}\left(h g Z \cap Y_{0}\right) \\
& =N(g)^{-\beta} \mu(Z),
\end{aligned}
$$

and the proof is complete.

Although the condition for a measure $v$ on $\Gamma \backslash Y$ to define a KMS-weight is easier to formulate in terms of the corresponding $\Gamma$-invariant measure on $Y$, it will also be important to work directly with $v$. For this we introduce the following operators on functions on $\Gamma \backslash X$. We shall often consider functions on $\Gamma \backslash X$ as $\Gamma$-invariant functions on $X$. 
Definition 2.5. Let $G$ act on a set $X$ and suppose $(G, \Gamma)$ is a Hecke a pair. The Hecke operator associated to $g \in G$ is the operator $T_{g}$ on $\Gamma$-invariant functions on $X$ defined by

$$
\left(T_{g} f\right)(x)=\frac{1}{R_{\Gamma}(g)} \sum_{h \in \Gamma \backslash \Gamma g \Gamma} f(h x)
$$

Clearly $T_{g} f$ is again $\Gamma$-invariant. It is not difficult to check that the map $\left[g^{-1}\right] \rightarrow R_{\Gamma}(g) T_{g}$ is a representation of the Hecke algebra $\mathscr{H}(G, \Gamma)$ on the space of $\Gamma$-invariant functions (notice that for $X=G$ this is exactly the way we defined the regular representation of $\mathscr{H}(G, \Gamma)$, so by decomposing an arbitrary $X$ into $G$-orbits one can obtain the general case without any computations).

The following three lemmas will be our main computational tools.

Lemma 2.6. Suppose $\mu$ is as in Proposition 2.1 and $v$ is the measure on $\Gamma \backslash Y$ determined by (2.2). Assume further that $Y=X$, the action of $G$ on $X$ is free and that $(G, \Gamma)$ is a Hecke pair with modular function $\Delta_{\Gamma}(g):=R_{\Gamma}\left(g^{-1}\right) / R_{\Gamma}(g)$. Then for any positive measurable function $f$ on $\Gamma \backslash X$ and $g \in G$ we have

$$
\int_{\Gamma \backslash X} T_{g} f d v=\Delta_{\Gamma}(g) N(g)^{\beta} \int_{\Gamma \backslash X} f d v
$$

Proof. Fix a point $x \in X$. We claim that there exists a neighbourhood $U$ of $x$ such that the sets $h U$ are disjoint for different $h$ in $\Gamma g^{-1} \Gamma$. Indeed, choose representatives $h_{1}, \ldots, h_{n}$ of the right $\Gamma$-cosets contained in $\Gamma g^{-1} \Gamma$. Since the action of $\Gamma$ is proper, there exists a neighbourhood $U$ of $x$ such that if $h_{i} U \cap \gamma h_{j} U \neq \varnothing$ for some $i, j$ and $\gamma \in \Gamma$ then $h_{i} x=\gamma h_{j} x$. But since the action of $G$ is free, the latter equality is possible only when $h_{i}=\gamma h_{j}$, so that $i=j$ and $\gamma=e$. Thus $h_{i} U \cap \gamma h_{j} U=\emptyset$ if $i \neq j$ or $\gamma \neq e$. Since $\Gamma g^{-1} \Gamma=\bigcup_{k=1}^{n} \Gamma h_{k}$, this proves the claim.

The set $\Gamma g^{-1} \Gamma U$ is therefore a disjoint union of the sets $h U, h \in \Gamma g^{-1} \Gamma$. So we can write

$$
\sum_{h \in \Gamma \backslash \Gamma g \Gamma} \mathbb{1}_{h^{-1} \Gamma U}=\mathbb{1}_{\Gamma g^{-1} \Gamma U}=\sum_{h \in \Gamma \backslash \Gamma g^{-1} \Gamma} \mathbb{1}_{\Gamma h U},
$$

Denoting by $p: X \mapsto \Gamma \backslash X$ the quotient map, we can rewrite the above in terms of functions on $\Gamma \backslash X$ as

$$
R_{\Gamma}(g) T_{g}\left(\mathbb{1}_{p(U)}\right)=\mathbb{1}_{p\left(\Gamma g^{-1} \Gamma U\right)}=\sum_{h \in \Gamma \backslash \Gamma g^{-1} \Gamma} \mathbb{1}_{p(h U)}
$$


It follows that

$$
\begin{aligned}
R_{\Gamma}(g) \int_{\Gamma \backslash X} T_{g}\left(\mathbb{1}_{p(U)}\right) d v & =\sum_{h \in \Gamma \backslash \Gamma g^{-1} \Gamma} v(p(h U)) \\
& =\sum_{h \in \Gamma \backslash \Gamma g^{-1} \Gamma} \mu(h U) \\
& =R_{\Gamma}\left(g^{-1}\right) N(g)^{\beta} v(p(U)) .
\end{aligned}
$$

In other words, the identity in the lemma holds for $f=\mathbb{1}_{p(U)}$. Since this is true for any $x$ and sufficiently small neighbourhood $U$ of $x$, we get the result.

Notice that by applying the above lemma to the characteristic function of $X$ we get the following: if a group $G$ acts freely on a space $X$ with a $G$-invariant measure $\mu$, and $\Gamma$ is an almost normal subgroup of $G$ (that is, $(G, \Gamma)$ is a Hecke pair) such that the action of $\Gamma$ on $X$ is proper and $0<\mu(\Gamma \backslash X)<\infty$, then $\Delta_{\Gamma}(g)=1$ for any $g \in G$. The same is true if we assume that the action of $G$ on $(X, \mu)$ is only essentially free.

Lemma 2.7. Suppose $\mu$ is as in Proposition 2.1 and $v$ is the measure on $\Gamma \backslash Y$ determined by (2.2). Assume the action of $G$ on $X$ is free and that $(G, \Gamma)$ is a Hecke pair. Assume further that $Y_{0}$ is a $\Gamma$-invariant measurable subset of $Y$ such that if $g Y_{0} \cap Y_{0} \neq \varnothing$ for some $g \in G$ then $g \in \Gamma$. Then for any $g \in G$ such that $g Y_{0} \subset Y$, measurable $Z \subset \Gamma \backslash Y_{0}$ and positive measurable function $f$ on $\Gamma \backslash Y$ we have

$$
\int_{\Gamma g Z} f d v=N(g)^{-\beta} R_{\Gamma}(g) \int_{Z} T_{g} f d v,
$$

where $\Gamma g Z=p\left(\Gamma g p^{-1}(Z)\right)$ and $p: X \rightarrow \Gamma \backslash X$ is the quotient map. In particular, $v(\Gamma g Z)=N(g)^{-\beta} R_{\Gamma}(g) v(Z)$.

Proof. Suppose $Z \subset \Gamma \backslash Y_{0}$ is measurable, and choose $U \subset Y_{0}$ measurable such that $Z=p(U)$ and $p$ is injective on $U$. For $g \in G$ let $h_{1}, \ldots, h_{n}$ be representatives of the right $\Gamma$-cosets contained in $\Gamma g \Gamma$. We claim that the map $p$ is injective on $h_{1} U, \ldots, h_{n} U$, and the images of these sets are disjoint. Indeed, assume $p\left(h_{i} x\right)=$ $p\left(h_{j} y\right)$ for some $i, j$ and $x, y \in U$, so that $\gamma h_{i} x=h_{j} y$ for some $\gamma \in \Gamma$. Since $U \subset Y_{0}$, our assumption on $Y_{0}$ implies $h_{j}^{-1} \gamma h_{i} \in \Gamma$. But then, since $p$ is injective on $U$, we get $x=y$, and since the action of $\Gamma$ is free, we conclude that $h_{j}^{-1} \gamma h_{i}=e$. It follows that $i=j$ and $h_{i} x=h_{j} y$, which proves the claim.

Furthermore, the union of the disjoint sets $p\left(h_{1} U\right), \ldots, p\left(h_{n} U\right)$ is the set 
$\Gamma g Z=p\left(\Gamma g p^{-1}(Z)\right)$. Hence, since $N\left(h_{i}\right)=N(g)$ for $i=1, \ldots, n$,

$$
\begin{aligned}
\int_{\Gamma g Z} f d \nu & =\sum_{i=1}^{n} \int_{h_{i} U} f \circ p d \mu \\
& =N(g)^{-\beta} \sum_{i=1}^{n} \int_{U} f\left(p\left(h_{i} \cdot\right)\right) d \mu \\
& =N(g)^{-\beta} R_{\Gamma}(g) \int_{Z} T_{g} f d \nu .
\end{aligned}
$$

The last assertion of the lemma follows by taking $f=\mathbb{1}_{\Gamma g Z}$ and observing that then $\left(T_{g} f\right)(z)=1$ for $z \in Z$.

To formulate the next lemma we introduce the following notation.

Definition 2.8. If $\beta \in \mathbb{R}$ and $S$ is a subsemigroup of $G$ containing $\Gamma$, then we define

$$
\zeta_{S, \Gamma}(\beta):=\sum_{s \in \Gamma \backslash S} N(s)^{-\beta}=\sum_{s \in \Gamma \backslash S / \Gamma} N(s)^{-\beta} R_{\Gamma}(s) .
$$

Lemma 2.9. Suppose $\mu$ is as in Proposition 2.1 and $v$ is the measure on $\Gamma \backslash Y$ determined by (2.2). Assume that the action of $G$ on $X$ is free and that $(G, \Gamma)$ is a Hecke pair. Assume further that $Y_{0}$ is a measurable $\Gamma$-invariant subset of $Y$, and $S$ a subsemigroup of $G$ containing $\Gamma$ such that

(i) if $g Y_{0} \cap Y_{0} \neq \emptyset$ for some $g \in G$ then $g \in \Gamma$;

(ii) $\bigcup_{s \in S} s Y_{0}$ is a subset of $Y$ of full measure;

(iii) $\zeta_{S, \Gamma}(\beta)<\infty$.

Let $H_{S}$ be the subspace of $S$-invariant functions in $L^{2}(\Gamma \backslash Y, v)$, that is, functions $f$ such that $f(y)=f(s y)$ for all $s \in S$ and a.a. $y \in Y$. Then

(1) if $f \in H_{S}$ then $\|f\|_{2}^{2}=\zeta_{S, \Gamma}(\beta) \int_{\Gamma \backslash Y_{0}}|f(t)|^{2} d v(t)$;

(2) the orthogonal projection $P: L^{2}(\Gamma \backslash Y, d \nu) \rightarrow H_{S}$ is given by

$$
\left.P f\right|_{S y}=\zeta_{S, \Gamma}(\beta)^{-1} \sum_{s \in \Gamma \backslash S / \Gamma} N(s)^{-\beta} R_{\Gamma}(s)\left(T_{s} f\right)(y) \text { for } y \in Y_{0} .
$$

Proof. By condition (i) the sets $\Gamma s Y_{0}$ are disjoint for $s$ in different double cosets of $\Gamma$. Since the union of such sets is the whole space $Y$ (modulo a set of measure zero), by Lemma 2.7 applied to $Z=\Gamma \backslash Y_{0}$ for any $f \in L^{2}(\Gamma \backslash Y, d \nu)$ we get

$$
\|f\|_{2}^{2}=\sum_{s \in \Gamma \backslash S / \Gamma} \int_{\Gamma s Z}|f|^{2} d v=\sum_{s \in \Gamma \backslash S / \Gamma} N(s)^{-\beta} R_{\Gamma}(s) \int_{\Gamma \backslash Y_{0}} T_{s}\left(|f|^{2}\right) d \nu .
$$


Since $T_{S}\left(|f|^{2}\right)=|f|^{2}$ for $f \in H_{S}$, this gives (1).

Turning to (2), denote by $T$ the operator on $L^{2}(\Gamma \backslash Y, d v)$ defined by the asserted formula for $P$. To see that it is well defined, notice first that the summation in the right hand side of (2.3) is finite for $f$ in the subspace of $L^{2}$-functions supported on a finite collection of sets of the form $p\left(s Y_{0}\right), s \in S$, which is a dense subspace of $L^{2}(\Gamma \backslash Y, d v)$. Thus the function $T f$ is well defined for $f$ in this subspace and, putting $\alpha_{s}=\zeta_{S, \Gamma}(\beta)^{-1} N(s)^{-\beta} R_{\Gamma}(s)$ and using (2.4) twice, we get

$$
\begin{aligned}
\|T f\|_{2}^{2} & =\zeta_{S, \Gamma}(\beta) \int_{\Gamma \backslash Y_{0}}|T f|^{2} d v \\
& \leq \zeta_{S, \Gamma}(\beta) \int_{\Gamma \backslash Y_{0}}\left(\sum_{s \in \Gamma \backslash S / \Gamma} \alpha_{s} T_{s}\left(|f|^{2}\right)\right) d v=\|f\|_{2}^{2} .
\end{aligned}
$$

It follows that $T$ extends to a well-defined contraction. Since $T f=f$ for $f \in H_{S}$, and the image of $T$ is $H_{S}$, we conclude that $T=P$.

\section{The Connes-Marcolli system}

Consider the group $G=\mathrm{GL}_{2}^{+}(\mathbb{Q})$ of invertible 2 by 2 matrices with rational coefficients and positive determinant, and its subgroup $\Gamma=\mathrm{SL}_{2}(\mathbb{Z})$. For a prime number $p$ consider the field $\mathbb{Q}_{p}$ of $p$-adic numbers and its compact subring $\mathbb{Z}_{p}$ of $p$-adic integers. We denote by $\mathbb{A}_{f}$ the space of finite adeles of $\mathbb{Q}$, that is, the restricted product of the fields of $\mathbb{Q}_{p}$ with respect to $\mathbb{Z}_{p}$, and by $\hat{\mathbb{Z}}=\prod_{p} \mathbb{Z}_{p}$ its maximal compact subring. The field $\mathbb{Q}$ is a subfield of $\mathbb{Q}_{p}$, so $\mathrm{GL}_{2}^{+}(\mathbb{Q})$ can be considered as a subgroup of $\mathrm{GL}_{2}\left(\mathbb{Q}_{p}\right)$. In particular, we have an action of $\mathrm{GL}_{2}^{+}(\mathbb{Q})$ on $\operatorname{Mat}_{2}\left(\mathbb{Q}_{p}\right)$ by multiplication on the left. Moreover, by considering the diagonal embedding of $\mathbb{Q}$ into $\mathbb{A}_{f}$ we get an embedding of $\mathrm{GL}_{2}^{+}(\mathbb{Q})$ into $\mathrm{GL}_{2}\left(\mathbb{A}_{f}\right)$, and thus an action of $\mathrm{GL}_{2}^{+}(\mathbb{Q})$ on $\operatorname{Mat}_{2}\left(\mathbb{A}_{f}\right)$. In addition $\mathrm{GL}_{2}^{+}(\mathbb{Q})$ acts by Möbius transformations on the upper halfplane $\mathbb{H}$. Therefore we have an action of $\mathrm{GL}_{2}^{+}(\mathbb{Q})$ on $\mathbb{U} \times \operatorname{Mat}_{2}\left(\mathbb{A}_{f}\right)$ such that for $g=\left(\begin{array}{ll}a & b \\ c & d\end{array}\right), \tau \in \mathbb{U}$ and $m=\left(m_{p}\right)_{p} \in \operatorname{Mat}_{2}\left(\mathbb{A}_{f}\right)$,

$$
g\left(\tau,\left(m_{p}\right)_{p}\right)=\left(\frac{a \tau+b}{c \tau+d},\left(g m_{p}\right)_{p}\right) .
$$

Note that the action of $\mathrm{SL}_{2}(\mathbb{Z})$ is proper, since already the action of $\mathrm{SL}_{2}(\mathbb{Z})$ on $\mathbb{H}$ is proper.

The $\mathrm{GL}_{2}$-system of Connes and Marcolli is now defined as follows, see [5], Section 1.8 .

Definition 3.1. The Connes-Marcolli algebra is the $C^{*}$-algebra $A=C_{\mathrm{r}}^{*}\left(\Gamma \backslash G \bigotimes_{\Gamma} Y\right)$, where $G=\mathrm{GL}_{2}^{+}(\mathbb{Q}), \Gamma=\mathrm{SL}_{2}(\mathbb{Z}), G$ acts diagonally on $X=\mathbb{U} \times \operatorname{Mat}_{2}\left(\mathbb{A}_{f}\right)$, 
and $Y=\mathbb{M} \times \mathrm{Mat}_{2}(\hat{\mathbb{Z}})$. The dynamics $\sigma$ on $A$ is defined by the homomorphism $N: \mathrm{GL}_{2}^{+}(\mathbb{Q}) \rightarrow \mathbb{R}_{+}^{*}, N(g)=\operatorname{det}(g)$.

Notice that since $\Gamma \backslash \mathbb{W}$ is not compact, the algebra $A$ is nonunital.

By [5], Lemma 1.28, the action of $\mathrm{GL}_{2}^{+}(\mathbb{Q})$ on $X \backslash(\mathbb{M} \times\{0\})$ is free. Recall briefly the reason. If $g m=m$ for some prime number $p$ and nonzero $m \in \operatorname{Mat}_{2}\left(\mathbb{Q}_{p}\right)$ then the spectrum of the matrix $g$ contains 1 , and hence $g$ is conjugate in $\mathrm{GL}_{2}^{+}(\mathbb{Q})$ to an upper-triangular matrix. But then $g$ has no fixed points in ㅁ․ Note that what we have actually shown is that the action of $\mathrm{GL}_{2}^{+}(\mathbb{Q})$ on $\mathbb{H} \times \operatorname{Mat}_{2}\left(\mathbb{Q}_{p}\right)^{\times}$, where $\operatorname{Mat}_{2}\left(\mathbb{Q}_{p}\right)^{\times}=\operatorname{Mat}_{2}\left(\mathbb{Q}_{p}\right) \backslash\{0\}$, is free for any prime number $p$.

Although the action of $\mathrm{GL}_{2}^{+}(\mathbb{Q})$ on $\mathbb{H} \times\{0\}$ is not free, this set can be ignored in the analysis of $\mathrm{KMS}_{\beta}$-states for $\beta \neq 0$, see the proof of [5, Proposition 1.30]. Again, recall briefly what happens. Consider the action of $G$ on $\tilde{X}=X \backslash(\mathbb{U} \times\{0\})$, put $\tilde{Y}=Y \backslash(\mathbb{H} \times\{0\}) \subset \tilde{X}$, and then define $I=C_{\mathrm{r}}^{*}\left(\Gamma \backslash G \bigotimes_{\Gamma} \tilde{Y}\right)$. Then $I$ can be considered as an ideal in $A$, and the quotient algebra $A / I$ is isomorphic to $C_{\mathrm{r}}^{*}\left(\Gamma \backslash G \times_{\Gamma} \mathbb{U}\right)$. Now if $\varphi$ is a $\sigma-\mathrm{KMS}_{\beta}$ state on $A$, the restriction $\left.\varphi\right|_{I}$ canonically extends to a KMS-functional on the multiplier algebra of $I$. Thus we get a KMS-functional $\tilde{\varphi} \leq \varphi$ on $A$. If $\tilde{\varphi} \neq \varphi$ then $\varphi-\tilde{\varphi}$ is a positive nonzero KMS-functional on $A$ which vanishes on $I$. Therefore we obtain a KMS-state on $A / I \cong C_{\mathrm{r}}^{*}\left(\Gamma \backslash G \times_{\Gamma} \mathbb{H}\right)$. By Lemma 1.3 the multiplier algebra of $C_{\mathrm{r}}^{*}\left(\Gamma \backslash G \times_{\Gamma} \mathbb{H}\right)$ contains the reduced Hecke $C^{*}$-algebra $C_{\mathrm{r}}^{*}(G, \Gamma)$. The latter algebra contains in turn the $C^{*}$-algebra of $Z(G) /(Z(G) \cap \Gamma)$, where $Z(G)$ is the center of $\mathrm{GL}_{2}^{+}(\mathbb{Q})$, that is, the group of scalar matrices. But since the dynamics scales nontrivially some unitaries in this algebra, the algebra can not have any $\mathrm{KMS}_{\beta}$-states for $\beta \neq 0$. This contradiction shows that $\varphi=\tilde{\varphi}$, so that $\varphi$ is completely determined by $\left.\varphi\right|_{I}$.

The action of $G$ on $\tilde{X}=\mathbb{H} \times \operatorname{Mat}_{2}\left(\mathbb{A}_{f}\right)^{\times}$, where $\operatorname{Mat}_{2}\left(\mathbb{A}_{f}\right)^{\times}=\operatorname{Mat}_{2}\left(\mathbb{A}_{f}\right) \backslash\{0\}$, is free, so we can apply Proposition 2.1 and conclude that there is a one-to-one correspondence between $\mathrm{KMS}_{\beta}$-weights on $I$ with domain of definition containing $C_{\mathrm{c}}(\Gamma \backslash \tilde{Y})$ and measures $\mu$ on $\tilde{Y}=\nVdash \times \operatorname{Mat}_{2}(\widehat{\mathbb{Z}})^{\times}$such that $\mu(g Z)=\operatorname{det}(g)^{-\beta} \mu(Z)$ if both $Z$ and $g Z$ are subsets of $\tilde{Y}$. By Lemma 2.2 we can uniquely extend any such measure to a measure on $\tilde{X}=G \tilde{Y}=\llbracket \times \operatorname{Mat}_{2}\left(\mathbb{A}_{f}\right)^{\times}$such that $\mu(g Z)=\operatorname{det}(g)^{-\beta} \mu(Z)$ for $Z \subset \tilde{X}$. To get a state on $I=C_{\mathrm{r}}^{*}\left(\Gamma \backslash G \bigotimes_{\Gamma} \tilde{Y}\right)$ we need the normalization condition $\mu(\Gamma \backslash \tilde{Y})=1$ (that is, the $\Gamma$-invariant measure $\mu$ on $\tilde{Y}$ defines a probability measure on $\Gamma \backslash \tilde{Y})$. Note also that if $\beta \neq 0$ and we have a measure on $X=\mathbb{H} \times \operatorname{Mat}_{2}\left(\mathbb{A}_{f}\right)$ with the same properties as above, then $\mathbb{H} \times \operatorname{Mat}_{2}\left(\mathbb{A}_{f}\right)^{\times}$is a subset of full measure, since scalar matrices act trivially on $\mathbb{U}$ and so $\mathbb{H}$ cannot support a measure scaled nontrivially by them.

Summarizing the above discussion we get the following.

Proposition 3.2. For $\beta \neq 0$ there is a one-to-one correspondence between $\sigma-K M S_{\beta}$ states on the Connes-Marcolli system and $\Gamma$-invariant measures $\mu$ on $\mathbb{W} \times \operatorname{Mat}_{2}\left(\mathbb{A}_{f}\right)$ 
such that

$$
\mu\left(\Gamma \backslash\left(\mathbb{H} \times \operatorname{Mat}_{2}(\widehat{\mathbb{Z}})\right)\right)=1 \text { and } \mu(g Z)=\operatorname{det}(g)^{-\beta} \mu(Z)
$$

for any $g \in \mathrm{GL}_{2}^{+}(\mathbb{Q})$ and compact $Z \subset \mathbb{U} \times \operatorname{Mat}_{2}\left(\mathbb{A}_{f}\right)$.

Denote by $\operatorname{Mat}_{2}^{i}\left(\mathbb{A}_{f}\right)$ the set of matrices $m=\left(m_{p}\right)_{p} \in \operatorname{Mat}_{2}\left(\mathbb{A}_{f}\right)$ such that $\operatorname{det}\left(m_{p}\right) \neq 0$ for every prime $p$. Notice that $\operatorname{Mat}_{2}^{i}\left(\mathbb{A}_{f}\right)$ is the set of non zero-divisors in $\operatorname{Mat}_{2}\left(\mathbb{A}_{f}\right)$. Our next goal is to show that if $\beta \neq 0,1$ then $\mathbb{H} \times \operatorname{Mat}_{2}^{i}\left(\mathbb{A}_{f}\right)$ is a subset of full measure for any measure $\mu$ as in Proposition 3.2.

First let us recall the following simple properties of the Hecke pair $(G, \Gamma)=$ $\left(\mathrm{GL}_{2}^{+}(\mathbb{Q}), \mathrm{SL}_{2}(\mathbb{Z})\right)$. Put $\mathrm{Mat}_{2}^{+}(\mathbb{Z})=\mathrm{GL}_{2}^{+}(\mathbb{Q}) \cap \mathrm{Mat}_{2}(\mathbb{Z})$.

Lemma 3.3. Every double coset of $\Gamma$ in $\mathrm{Mat}_{2}^{+}(\mathbb{Z})$ has a unique representative of the form $\left(\begin{array}{ll}a & 0 \\ 0 & d\end{array}\right)$ with $a, d \in \mathbb{N}$ and $a \mid d$. Furthermore,

$$
R_{\Gamma}\left(\begin{array}{ll}
a & 0 \\
0 & d
\end{array}\right)=\frac{d}{a} \prod_{p \text { prime: } p a \mid d}\left(1+p^{-1}\right)
$$

and as representatives of the right cosets of $\Gamma$ contained in $\Gamma\left(\begin{array}{ll}a & 0 \\ 0 & d\end{array}\right) \Gamma$ we can take the matrices

$$
\left(\begin{array}{cc}
a k & a m \\
0 & a l
\end{array}\right)
$$

with $k, l \in \mathbb{N}$ and $m \in \mathbb{Z}$ such that $k l=d / a, 0 \leq m<l$ and $\operatorname{gcd}(k, l, m)=1$.

In particular, $R_{\Gamma}(g)=R_{\Gamma}\left(g^{-1}\right)$ for every $g \in \mathrm{GL}_{2}^{+}(\mathbb{Q})$.

Proof. See e.g. [13], Chapter IV.

For a prime $p$ put $G_{p}=\mathrm{GL}_{2}^{+}\left(\mathbb{Z}\left[p^{-1}\right]\right) \subset \mathrm{GL}_{2}^{+}(\mathbb{Q})$. Observe that if $g \in G_{p}$ then $\operatorname{det}(g)$ is a power of $p$, and if we multiply $g$ by a sufficiently large power of $\left(\begin{array}{ll}p & 0 \\ 0 & p\end{array}\right)$, we get an element in $\mathrm{Mat}_{2}^{+}(\mathbb{Z})$ with determinant a power of $p$. But by Lemma 3.3 the double coset of $\Gamma$ containing such an element has a representative of the form $\left(\begin{array}{cc}p^{k} & 0 \\ 0 & p^{l}\end{array}\right), 0 \leq k \leq l$. We may therefore conclude that $G_{p}$ is the subgroup of $\mathrm{GL}_{2}^{+}(\mathbb{Q})$ generated by $\Gamma$ and $\left(\begin{array}{ll}1 & 0 \\ 0 & p\end{array}\right)$. Using that a positive rational number is a power of $p$ if and only if it belongs to the group of units $\mathbb{Z}_{q}^{*}$ of the ring $\mathbb{Z}_{q}$ for all primes $q \neq p$, we may also conclude that $g \in \mathrm{GL}_{2}^{+}(\mathbb{Q})$ belongs to $G_{p}$ if and only if it belongs to $\mathrm{GL}_{2}\left(\mathbb{Z}_{q}\right)$ for all $q \neq p$.

Lemma 3.4. We have $\mathrm{GL}_{2}\left(\mathbb{Q}_{p}\right)=G_{p} \mathrm{GL}_{2}\left(\mathbb{Z}_{p}\right)$. 
Proof. Let $r \in \mathrm{GL}_{2}\left(\mathbb{Q}_{p}\right)$. Then $r \mathbb{Z}_{p}^{2}$ is a $\mathbb{Z}_{p}$-lattice in $\mathbb{Q}_{p}^{2}$, that is, an open compact $\mathbb{Z}_{p}$-submodule. By [22], Theorem V.2, there exists a subgroup $L \cong \mathbb{Z}^{2}$ of $\mathbb{Q}^{2}$ such that the closure of $L$ in $\mathbb{Q}_{p}^{2}$ coincides with $r \mathbb{Z}_{p}^{2}$, and the closure of $L$ in $\mathbb{Q}_{q}^{2}$ is $\mathbb{Z}_{q}^{2}$ for $q \neq p$. Choose $g \in \mathrm{GL}_{2}^{+}(\mathbb{Q})$ such that $g \mathbb{Z}^{2}=L$. Since $g \mathbb{Z}_{p}^{2}=r \mathbb{Z}_{p}^{2}$, we have $g^{-1} r \in \mathrm{GL}_{2}\left(\mathbb{Z}_{p}\right)$. Since $g \mathbb{Z}_{q}^{2}=\mathbb{Z}_{q}^{2}$ for $q \neq p$, we also have $g \in \mathrm{GL}_{2}\left(\mathbb{Z}_{q}\right)$. Hence $g \in G_{p}$.

It is also possible to give an elementary proof of Lemma 3.4 using matrix factorization and density of $\mathbb{Z}\left[p^{-1}\right]$ in $\mathbb{Q}_{p}$.

Lemma 3.5. Let $p$ be a prime and $\mu_{p}$ a $\Gamma$-invariant measure on $\mathbb{M} \times \operatorname{Mat}_{2}\left(\mathbb{Q}_{p}\right)$ such that $\mu_{p}(\mathbb{W} \times\{0\})=0, \mu_{p}\left(\Gamma \backslash\left(\mathbb{W} \times \operatorname{Mat}_{2}\left(\mathbb{Z}_{p}\right)\right)\right)<\infty$ and $\mu_{p}(g Z)=$ $\operatorname{det}(g)^{-\beta} \mu_{p}(Z)$ for $g \in G_{p}$ and $Z \subset \mathbb{W} \times \operatorname{Mat}_{2}\left(\mathbb{Q}_{p}\right)$. If $\beta \neq 1$, then the set $\mathbb{W} \times \mathrm{GL}_{2}\left(\mathbb{Q}_{p}\right)$ is a subset of full measure in $\mathbb{U} \times \operatorname{Mat}_{2}\left(\mathbb{Q}_{p}\right)$.

Proof. Denote by $\tilde{v}$ the measure on $\Gamma \backslash\left(\mathbb{M} \times \operatorname{Mat}_{2}\left(\mathbb{Q}_{p}\right)\right)$ defined by the $\Gamma$-invariant measure $\mu_{p}$. For a $\Gamma$-invariant subset $Z$ of $\operatorname{Mat}_{2}\left(\mathbb{Q}_{p}\right)$, the set $\mathbb{U} \times Z$ is $\Gamma$-invariant. We can thus define a measure $v$ on the $\sigma$-algebra of $\Gamma$-invariant Borel subsets of $\operatorname{Mat}_{2}\left(\mathbb{Q}_{p}\right)$ by $v(Z)=\tilde{v}(\Gamma \backslash(\mathbb{H} \times Z))$. Note that since the action of $\Gamma$ on $\operatorname{Mat}_{2}\left(\mathbb{Q}_{p}\right)$ is not proper and, accordingly, the quotient space $\Gamma \backslash \operatorname{Mat}_{2}\left(\mathbb{Q}_{p}\right)$ is quite bad, we do not want to consider $\Gamma$-invariant subsets of $\operatorname{Mat}_{2}\left(\mathbb{Q}_{p}\right)$ as subsets of this quotient space, and do not try to define a measure on all Borel subsets of $\operatorname{Mat}_{2}\left(\mathbb{Q}_{p}\right)$ out of $v$.

If $g \in G_{p}$ and $f$ is a positive Borel $\Gamma$-invariant function on $\operatorname{Mat}_{2}\left(\mathbb{Q}_{p}\right)$ then by Lemma 2.6 applied to the function $F:(\tau, m) \mapsto f(m)$ on $\Gamma \backslash\left(\mathbb{M} \times \operatorname{Mat}_{2}\left(\mathbb{Q}_{p}\right)\right)$ we conclude that

$$
\begin{aligned}
\int_{\operatorname{Mat}_{2}\left(\mathbb{Q}_{p}\right)} T_{g} f d v & =\int_{\Gamma \backslash\left(\mathbb{Q} \times \operatorname{Mat}_{2}\left(\mathbb{Q}_{p}\right)\right)} T_{g} F d \tilde{v} \\
& =\operatorname{det}(g)^{\beta} \int_{\Gamma \backslash\left(\mathbb{T} \times \operatorname{Mat}_{2}\left(\mathbb{Q}_{p}\right)\right)} F d \tilde{v}=\operatorname{det}(g)^{\beta} \int_{\operatorname{Mat}_{2}\left(\mathbb{Q}_{p}\right)} f d v .
\end{aligned}
$$

By assumption we also have $v\left(\operatorname{Mat}_{2}\left(\mathbb{Z}_{p}\right)\right)<\infty$. We have to show that the measure of the set of nonzero singular matrices is zero.

We claim that the set of nonzero singular matrices with coefficients in $\mathbb{Q}_{p}$ is the disjoint union of the sets

$$
Z_{k}=\operatorname{SL}_{2}\left(\mathbb{Z}_{p}\right)\left(\begin{array}{cc}
0 & 0 \\
0 & p^{k}
\end{array}\right) \operatorname{GL}_{2}\left(\mathbb{Z}_{p}\right), \quad k \in \mathbb{Z} .
$$

This is proved in a standard way: given a nonzero singular matrix we use multiplication by elements of $\mathrm{GL}_{2}\left(\mathbb{Z}_{p}\right)$ on the right to get a matrix with zero first column, and then multiplication by elements of $\mathrm{SL}_{2}\left(\mathbb{Z}_{p}\right)$ on the left to get the required form. To show that the sets do not intersect, observe that the maximum of the $p$-adic valuations of the 
coefficients of a matrix does not change under multiplication by elements of $\mathrm{GL}_{2}\left(\mathbb{Z}_{p}\right)$ on either side.

Consider the functions $f_{k}=\mathbb{1}_{Z_{k}}, k \in \mathbb{Z}$. For $g=\left(\begin{array}{ll}1 & 0 \\ 0 & p^{-1}\end{array}\right)$ we claim that

$$
T_{g} f_{0}=\frac{1}{p+1} f_{0}+\frac{p}{p+1} f_{1} .
$$

Indeed, since the action of $G_{p}$ commutes with the right action of $\mathrm{GL}_{2}\left(\mathbb{Z}_{p}\right)$, the function $T_{g} f_{0}$ is $\mathrm{GL}_{2}\left(\mathbb{Z}_{p}\right)$-invariant. On the other hand, the sets $Z_{k}$ are clopen subsets of the set of singular matrices, so that the function $f_{0}$ is continuous on this set. But then $T_{g} f_{0}$ is also continuous. Since $T_{g} f_{0}$ is $\Gamma$-invariant, and $\Gamma$ is dense in $\mathrm{SL}_{2}\left(\mathbb{Z}_{p}\right)$ (see e.g. [20], Lemma 1.38, for an elementary proof of a stronger result: $\Gamma$ is dense in $\mathrm{SL}_{2}(\widehat{\mathbb{Z}})$ ), we conclude that $T_{g} f_{0}$ is left $\mathrm{SL}_{2}\left(\mathbb{Z}_{p}\right)$-invariant. Hence $T_{g} f_{0}$ is constant on the sets $Z_{k}$. So to prove the above identity it suffices to check it on the matrices $\left(\begin{array}{cc}0 & 0 \\ 0 & p^{k}\end{array}\right)$. Since $g=\left(\begin{array}{cc}p^{-1} & 0 \\ 0 & p^{-1}\end{array}\right)\left(\begin{array}{ll}p & 0 \\ 0 & 1\end{array}\right)$, by Lemma 3.3 we can take the matrices

$$
\left(\begin{array}{cc}
1 & 0 \\
0 & p^{-1}
\end{array}\right), \quad\left(\begin{array}{cc}
p^{-1} & n p^{-1} \\
0 & 1
\end{array}\right), \quad 0 \leq n \leq p-1,
$$

as representatives of the right cosets of $\Gamma$ contained in $\Gamma g \Gamma$. Then

$$
\left(T_{g} f_{0}\right)\left(\begin{array}{cc}
0 & 0 \\
0 & p^{-k}
\end{array}\right)=\frac{1}{p+1} f_{0}\left(\begin{array}{cc}
0 & 0 \\
0 & p^{k-1}
\end{array}\right)+\frac{1}{p+1} \sum_{n=0}^{p-1} f_{0}\left(\begin{array}{cc}
0 & n p^{k-1} \\
0 & p^{k}
\end{array}\right) .
$$

Since the matrices $\left(\begin{array}{cc}0 & 0 \\ 0 & p^{-k}\end{array}\right)$ and $\left(\begin{array}{cc}0 & n p^{k-1} \\ 0 & p^{k}\end{array}\right), 1 \leq n \leq p-1$, belong to $Z_{k-1}$, we see that

$$
\left.T_{g} f_{0}\right|_{Z_{1}}=\frac{p}{p+1},\left.\quad T_{g} f_{0}\right|_{Z_{0}}=\frac{1}{p+1},\left.\quad T_{g} f_{0}\right|_{Z_{k}}=0 \text { for } k \neq 0,1,
$$

and this is exactly what we claimed.

It follows from (3.1) that

$$
p^{-\beta} v\left(Z_{0}\right)=\frac{1}{p+1} v\left(Z_{0}\right)+\frac{p}{p+1} v\left(Z_{1}\right) .
$$

On the other hand, for $g=\left(\begin{array}{cc}p^{-1} & 0 \\ 0 & p^{-1}\end{array}\right)$ we get $T_{g} f_{k}=f_{k+1}$, so that

$$
p^{-2 \beta} v\left(Z_{k}\right)=v\left(Z_{k+1}\right) \text {. }
$$

If $v\left(Z_{0}\right) \neq 0$ this implies that $p^{-\beta}$ is a solution of the quadratic equation

$$
(p+1) x=1+p x^{2},
$$


Thus either $p^{-\beta}=p^{-1}$ or $p^{-\beta}=1$. Since $\beta \neq 1$ we get $\beta=0$. But then $v\left(Z_{k}\right)=v\left(Z_{0}\right)$ for any $k$, and this contradicts $v\left(\operatorname{Mat}_{2}\left(\mathbb{Z}_{p}\right)\right)<\infty$. The contradiction shows that $v\left(Z_{0}\right)=0$. Hence $v\left(Z_{k}\right)=0$ for any $k$, and we conclude that the measure of the set of singular matrices is zero.

We are now ready to show that for $\beta \neq 0,1$ the set $\operatorname{Mat}_{2}\left(\mathbb{A}_{f}\right) \backslash \operatorname{Mat}_{2}^{i}\left(\mathbb{A}_{f}\right)$ of zero-divisors has measure zero.

Corollary 3.6. Assume $\beta \neq 0,1$ and $\mu$ is a measure with properties as in Proposition 3.2. Then $\mathbb{M} \times \operatorname{Mat}_{2}^{i}\left(\mathbb{A}_{f}\right)$ is a subset of full measure in $\mathbb{M} \times \operatorname{Mat}_{2}\left(\mathbb{A}_{f}\right)$.

Proof. Fix a prime $p$. First of all note that the set

$$
\left\{(\tau, m) \in \mathbb{M} \times \operatorname{Mat}_{2}\left(\mathbb{A}_{f}\right) \mid m_{p}=0\right\}
$$

has measure zero. Indeed, as we already remarked before Proposition 3.2, the set $\mathbb{H} \times\{0\}$ has measure zero. So if our claim is not true, the set

$$
\left\{(\tau, m) \in \mathbb{W} \times \operatorname{Mat}_{2}(\widehat{\mathbb{Z}})^{\times} \mid m_{p}=0\right\}
$$

has positive measure. Since the action of $\Gamma$ on this set is free, there is a subset $U$ of positive measure such that $\gamma U \cap U=\varnothing$ for $\gamma \in \Gamma, \gamma \neq e$. Then for $g=\left(\begin{array}{ll}p & 0 \\ 0 & p\end{array}\right)$ the set $U_{k}=g^{k} U, k \in \mathbb{Z}$, still has the property that $\gamma U_{k} \cap U_{k}=\emptyset$ for $\gamma \in \Gamma$, $\gamma \neq e$, since $g$ commutes with $\Gamma$. As $U_{k}$ is contained in $\mathbb{W} \times \operatorname{Mat}_{2}(\widehat{\mathbb{Z}})$, it follows that $\mu\left(U_{k}\right) \leq 1$. On the other hand, $\mu\left(U_{k}\right)=p^{-2 \beta k} \mu(U)$. Letting $k \rightarrow-\infty$ if $\beta>0$ and $k \rightarrow+\infty$ if $\beta<0$, we get a contradiction.

Consider now the restriction of $\mu$ to the set

$$
\nVdash \times \operatorname{Mat}_{2}\left(\mathbb{Q}_{p}\right) \times \prod_{q \neq p} \operatorname{Mat}_{2}\left(\mathbb{Z}_{q}\right),
$$

and use the projection onto the first two factors to get a measure $\mu_{p}$ on $\mathbb{M} \times \operatorname{Mat}_{2}\left(\mathbb{Q}_{p}\right)$. By the first part of the proof the set $\mathbb{W} \times\{0\}$ has $\mu_{p}$-measure zero. Since the image of $G_{p}$ in $\mathrm{GL}_{2}\left(\mathbb{Q}_{q}\right)$ lies in $\mathrm{GL}_{2}\left(\mathbb{Z}_{q}\right)$ for $q \neq p$, the scaling property of $\mu$ implies that

$$
\mu_{p}(g Z)=\operatorname{det}(g)^{-\beta} \mu_{p}(Z) \text { for } Z \subset \mathbb{M} \times \operatorname{Mat}_{2}\left(\mathbb{Q}_{p}\right), g \in G_{p} .
$$

Since the action of $\Gamma$ on $\mathbb{U} \times \operatorname{Mat}_{2}\left(\mathbb{Q}_{p}\right)^{\times}$is free, the normalization condition on $\mu$ implies that $\mu_{p}\left(\Gamma \backslash\left(\mathbb{M} \times \operatorname{Mat}_{2}\left(\mathbb{Z}_{p}\right)\right)=1\right.$. Thus $\mu_{p}$ satisfies the assumptions of Lemma 3.5. Hence $\mathbb{H} \times \mathrm{GL}_{2}\left(\mathbb{Q}_{p}\right)$ is a set of full $\mu_{p}$-measure. This means that the set of points $(\tau, m) \in \mathbb{H} \times \operatorname{Mat}_{2}(\hat{\mathbb{Z}})$ with $\operatorname{det}\left(m_{p}\right)=0$ has $\mu$-measure zero. By taking the union of such sets for all primes $p$ and multiplying it by elements of $\mathrm{GL}_{2}^{+}(\mathbb{Q})$ we get a set of measure zero, which is the complement of the set $\llbracket \times \operatorname{Mat}_{2}^{i}\left(\mathbb{A}_{f}\right)$. 
To get further properties of a measure $\mu$ as above, let us recall the following wellknown computation, see e.g. [20], Section 3.2, for more general results on formal Dirichlet series. Denote by $S_{p}$ the semigroup $G_{p} \cap \operatorname{Mat}_{2}^{+}(\mathbb{Z})$. Alternatively, $S_{p}$ is the set of elements $m \in \operatorname{Mat}_{2}^{+}(\mathbb{Z})$ with determinant a nonnegative power of $p$. Then from Lemma 3.3 we know that as representatives of the right cosets of $\Gamma$ in $S_{p}$ we can take the matrices $\left(\begin{array}{cc}p^{k} & m \\ 0 & p^{l}\end{array}\right), k, l \geq 0,0 \leq m<p^{l}$. Therefore

$$
\begin{aligned}
\zeta_{S_{p}, \Gamma}(\beta) & =\sum_{s \in \Gamma \backslash S_{p}} \operatorname{det}(s)^{-\beta}=\sum_{k, l=0}^{\infty} p^{-\beta(k+l)} p^{l} \\
& = \begin{cases}+\infty & \text { if } \beta \leq 1, \\
\left(1-p^{-\beta}\right)^{-1}\left(1-p^{-\beta+1}\right)^{-1} & \text { if } \beta>1 .\end{cases}
\end{aligned}
$$

Since $\Gamma=G_{p} \cap \mathrm{GL}_{2}\left(\mathbb{Z}_{p}\right)$, we can apply Lemma 2.7 to the group $G_{p}$ acting on $\mathbb{M} \times \operatorname{Mat}_{2}\left(\mathbb{A}_{f}\right)^{\times}$and the set

$$
Y_{0}=\mathbb{U} \times \mathrm{GL}_{2}\left(\mathbb{Z}_{p}\right) \times \prod_{q \neq p} \operatorname{Mat}_{2}\left(\mathbb{Z}_{q}\right)
$$

Then for any $s \in S_{p}$ we get

$$
\mu\left(\Gamma \backslash \Gamma s Y_{0}\right)=\operatorname{det}(s)^{-\beta} R_{\Gamma}(s) \mu\left(\Gamma \backslash Y_{0}\right) .
$$

The sets $\Gamma s Y_{0}$ are disjoint for $s$ in different double cosets of $\Gamma$, and their union is the set

$$
\mathbb{U} \times \operatorname{Mat}_{2}^{i}\left(\mathbb{Z}_{p}\right) \times \prod_{q \neq p} \operatorname{Mat}_{2}\left(\mathbb{Z}_{q}\right),
$$

where $\operatorname{Mat}_{2}^{i}\left(\mathbb{Z}_{p}\right)=\operatorname{Mat}_{2}\left(\mathbb{Z}_{p}\right) \cap \mathrm{GL}_{2}\left(\mathbb{Q}_{p}\right)$. By Corollary 3.6 the above set is a subset of $\mathbb{Z} \times \mathrm{Mat}_{2}(\hat{\mathbb{Z}})$ of full measure for $\beta \neq 0,1$. Therefore we obtain

$$
\begin{aligned}
1 & =\sum_{s \in \Gamma \backslash S_{p} / \Gamma} \mu\left(\Gamma \backslash \Gamma s Y_{0}\right)=\sum_{s \in \Gamma \backslash S_{p} / \Gamma} \operatorname{det}(s)^{-\beta} R_{\Gamma}(s) \mu\left(\Gamma \backslash Y_{0}\right) \\
& =\zeta_{S_{p}, \Gamma}(\beta) \mu\left(\Gamma \backslash Y_{0}\right) .
\end{aligned}
$$

This gives a contradiction if $\beta<1$. Thus for $\beta<1, \beta \neq 0$, there are no $\mathrm{KMS}_{\beta}$-states. On the other hand, for $\beta>1$ we get

$$
\mu\left(\Gamma \backslash Y_{0}\right)=\zeta_{S_{p}, \Gamma}(\beta)^{-1}=\left(1-p^{-\beta}\right)\left(1-p^{-\beta+1}\right) .
$$

Assuming now that $\beta>1$ we can perform a similar computation for any finite set of primes instead of just one prime. Given a finite set $F$ of primes consider the group 
$G_{F}$ generated by $G_{p}$ for all $p \in F$. Put also $S_{F}=\operatorname{Mat}_{2}^{+}(\mathbb{Z}) \cap G_{F}$. Then $S_{F}$ is the set of matrices $m \in \operatorname{Mat}_{2}^{+}(\mathbb{Z})$ such that all prime divisors of $\operatorname{det}(m)$ belong to $F$. Let

$$
Y_{F}=\mathbb{U} \times\left(\prod_{p \in F} \mathrm{GL}_{2}\left(\mathbb{Z}_{p}\right)\right) \times\left(\prod_{q \notin F} \operatorname{Mat}_{2}\left(\mathbb{Z}_{q}\right)\right) .
$$

Then a computation similar to (3.2) and (3.3) yields

$$
\begin{aligned}
& \zeta_{S_{F}, \Gamma}(\beta)=\prod_{p \in F}\left(1-p^{-\beta}\right)^{-1}\left(1-p^{-\beta+1}\right)^{-1} \text { and } \\
& \mu\left(\Gamma \backslash Y_{F}\right)=\prod_{p \in F}\left(1-p^{-\beta}\right)\left(1-p^{-\beta+1}\right) .
\end{aligned}
$$

The intersection of the sets $Y_{F}$ over all finite subsets $F$ of prime numbers is the set $\mathbb{U} \times \mathrm{GL}_{2}(\widehat{\mathbb{Z}})$. So for $\beta>2$ we get

$$
\mu\left(\Gamma \backslash\left(\mathbb{T} \times \mathrm{GL}_{2}(\widehat{\mathbb{Z}})\right)\right)=\prod_{p}\left(1-p^{-\beta}\right)\left(1-p^{-\beta+1}\right)=\zeta(\beta)^{-1} \zeta(\beta-1)^{-1},
$$

where $\zeta$ is the Riemann $\zeta$-function. On the other hand, for $\beta \in(1,2]$ we obtain that

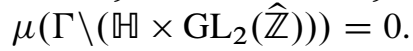

Assume now that $\beta>2$. In this case similarly to (3.2) we have

$$
\zeta_{\mathrm{Mat}_{2}^{+}(\mathbb{Z}), \Gamma}(\beta)=\zeta(\beta) \zeta(\beta-1) .
$$

So analogously to (3.3) we get

$$
\mu\left(\Gamma \backslash \mathrm{Mat}_{2}^{+}(\mathbb{Z})\left(\mathbb{W} \times \mathrm{GL}_{2}(\hat{\mathbb{Z}})\right)\right)=\zeta_{\mathrm{Mat}_{2}^{+}(\mathbb{Z}), \Gamma}(\beta) \mu\left(\Gamma \backslash\left(\mathbb{W} \times \mathrm{GL}_{2}(\hat{\mathbb{Z}})\right)\right)=1 .
$$

We thus see that $\operatorname{Mat}_{2}^{+}(\mathbb{Z})\left(\mathbb{W} \times \mathrm{GL}_{2}(\hat{\mathbb{Z}})\right)$ is a subset of $\mathbb{U} \times \operatorname{Mat}_{2}(\hat{\mathbb{Z}})$ of full measure. Hence $\mathrm{GL}_{2}^{+}(\mathbb{Q})\left(\mathbb{W} \times \mathrm{GL}_{2}(\widehat{\mathbb{Z}})\right)$ is a subset of $\mathbb{U} \times \operatorname{Mat}_{2}\left(\mathbb{A}_{f}\right)$ of full measure. By Lemma 3.4 the set $\mathrm{GL}_{2}^{+}(\mathbb{Q})\left(\mathbb{W} \times \mathrm{GL}_{2}(\hat{\mathbb{Z}})\right)$ is nothing but $\mathbb{U} \times \mathrm{GL}_{2}\left(\mathbb{A}_{f}\right)$.

To summarize, we have shown that for $\beta>2$ the problem of finding all measures $\mu$ on $\mathbb{U} \times \operatorname{Mat}_{2}\left(\mathbb{A}_{f}\right)$ satisfying the conditions in Proposition 3.2 reduces to finding all measures on $\mathbb{\square} \times \mathrm{GL}_{2}\left(\mathbb{A}_{f}\right)$ such that

$$
\mu(g Z)=\operatorname{det}(g)^{-\beta} \mu(Z) \text { and } \mu\left(\Gamma \backslash\left(\mathbb{H} \times \mathrm{GL}_{2}(\widehat{\mathbb{Z}})\right)\right)=\zeta(\beta)^{-1} \zeta(\beta-1)^{-1} .
$$

By Lemma 2.4 any $\Gamma$-invariant measure on $\mathbb{U} \times \mathrm{GL}_{2}(\hat{\mathbb{Z}})$ extends uniquely to a measure on $\mathbb{U} \times \mathrm{GL}_{2}\left(\mathbb{A}_{f}\right)$ satisfying the scaling condition. Thus we get a one-to-one correspondence between measures $\mu$ with properties as in Proposition 3.2 and measures on $\Gamma \backslash\left(\mathbb{Q} \times \mathrm{GL}_{2}(\hat{\mathbb{Z}})\right)$ of total mass $\zeta(\beta)^{-1} \zeta(\beta-1)^{-1}$. Clearly, extremal measures $\mu$ correspond to point masses.

We have thus recovered the following result of Connes and Marcolli [5], Theorem 1.26 and Corollary 1.32 . 
Theorem 3.7. For the Connes-Marcolli $\mathrm{GL}_{2}$-system we have:

(i) for $\beta \in(-\infty, 0) \cup(0,1)$ there are no $K M S_{\beta}$-states;

(ii) for $\beta>2$ there is a one-to-one affine correspondence between $K M S_{\beta}$-states and probability measures on $\Gamma \backslash\left(\mathbb{H} \times \mathrm{GL}_{2}(\widehat{\mathbb{Z}})\right)$; in particular, extremal KMS $S_{\beta}$-states are in bijection with $\Gamma$-orbits in $\mathbb{H} \times \mathrm{GL}_{2}(\widehat{\mathbb{Z}})$.

Remark 3.8. This is not exactly what is stated in [5]. First of all, the cases $\beta=0,1$ require considerations with singular matrices, and in these cases we do have KMSstates, see Remark 4.8 below. Secondly, the classification of extremal $\mathrm{KMS}_{\beta}$-states for $\beta>2$ in [5], Theorem 1.26, is in terms of invertible $\mathbb{Q}$-lattices up to scaling. To see that our Theorem 3.7(ii) says the same, recall that the isomorphism from [5], equation (1.87), identifies $\Gamma \backslash\left(\mathbb{H} \times \mathrm{GL}_{2}(\hat{\mathbb{Z}})\right)$ with the set of invertible $\mathbb{Q}$-lattices in $\mathbb{C}$ up to scaling, and observe that the state $\varphi_{\beta, l}$ defined in [5], Theorem 1.26(ii), associated with $l=(\tau, \rho) \in \mathbb{U} \times \mathrm{GL}_{2}(\widehat{\mathbb{Z}})$ is exactly the $\mathrm{KMS}_{\beta}$-state corresponding to the orbit $\Gamma(\tau, \rho)$. Since the $\mathbb{Q}$-lattice picture will not be used in the remaining part of the paper, we omit the details.

\section{Uniqueness of the $\mathrm{KMS}_{\beta}$-state in the critical region $1<\beta \leq 2$}

In this section we analyze the Connes-Marcolli system in the region $\beta \in(1,2]$.

For each such $\beta$ let us first construct a $\mathrm{KMS}_{\beta}$-state, or equivalently, a measure $\mu_{\beta}$ on $\mathbb{H} \times \operatorname{Mat}_{2}\left(\mathbb{A}_{f}\right)$ satisfying the conditions in Proposition 3.2.

For each prime number $p$ consider the Haar measure on $\mathrm{GL}_{2}\left(\mathbb{Z}_{p}\right)$ normalized such that the total mass is $\left(1-p^{-\beta}\right)\left(1-p^{-\beta+1}\right)$. By the same argument as in the proof of Lemma 2.4 , this measure extends to a unique measure $\mu_{\beta, p}$ on $\operatorname{GL}_{2}\left(\mathbb{Q}_{p}\right)$ such that

$$
\mu_{\beta, p}(Z)=\sum_{g \in \mathrm{GL}_{2}\left(\mathbb{Z}_{p}\right) \backslash \mathrm{GL}_{2}\left(\mathbb{Q}_{p}\right)}|\operatorname{det}(g)|_{p}^{-\beta} \mu_{\beta, p}\left(g Z \cap \mathrm{GL}_{2}\left(\mathbb{Z}_{p}\right)\right)
$$

for compact $Z \subset \mathrm{GL}_{2}\left(\mathbb{Q}_{p}\right)$, where $|a|_{p}$ denotes the $p$-adic valuation of $a$. The measure $\mu_{\beta, p}$ satisfies

$$
\mu_{\beta, p}(g Z)=|\operatorname{det}(g)|_{p}^{\beta} \mu_{\beta, p}(Z) \quad \text { for } g \in \mathrm{GL}_{2}\left(\mathbb{Q}_{p}\right) .
$$

Since $|\operatorname{det}(g)|_{p}=1$ for $g \in \mathrm{GL}_{2}\left(\mathbb{Z}_{p}\right)$, it is clear that $\mu_{\beta, p}$ is left $\mathrm{GL}_{2}\left(\mathbb{Z}_{p}\right)$-invariant. But since the Haar measure on $\mathrm{GL}_{2}\left(\mathbb{Z}_{p}\right)$ is biinvariant, we conclude that $\mu_{\beta, p}$ is also right $\mathrm{GL}_{2}\left(\mathbb{Z}_{p}\right)$-invariant. By setting $\mu_{\beta, p}(Z)=\mu_{\beta, p}\left(Z \cap \mathrm{GL}_{2}\left(\mathbb{Q}_{p}\right)\right)$ for Borel $Z \subset \operatorname{Mat}_{2}\left(\mathbb{Q}_{p}\right)$ we extend $\mu_{\beta, p}$ to a measure on $\operatorname{Mat}_{2}\left(\mathbb{Q}_{p}\right)$. Using that $\operatorname{Mat}_{2}^{i}\left(\mathbb{Z}_{p}\right)=$ $S_{p} \mathrm{GL}_{2}\left(\mathbb{Z}_{p}\right)$, similarly to (3.3) we find

$$
\mu_{\beta, p}\left(\operatorname{Mat}_{2}\left(\mathbb{Z}_{p}\right)\right)=\zeta_{S_{p}, \Gamma}(\beta) \mu_{\beta, p}\left(\mathrm{GL}_{2}\left(\mathbb{Z}_{p}\right)\right)=1
$$


Hence we can define a measure on $\operatorname{Mat}_{2}\left(\mathbb{A}_{f}\right)$ by $\mu_{\beta, f}=\prod_{p} \mu_{\beta, p}$. By construction and Lemma 3.5 this is the unique product-measure such that $\mu_{\beta, f}\left(\operatorname{Mat}_{2}(\widehat{\mathbb{Z}})\right)=1$ and

$$
\mu_{\beta, f}(g Z r)=\left(\prod_{p}\left|\operatorname{det}\left(g_{p}\right)\right|_{p}\right)^{\beta} \mu_{\beta, f}(Z)
$$

for $Z \subset \operatorname{Mat}_{2}\left(\mathbb{A}_{f}\right), g=\left(g_{p}\right)_{p} \in \mathrm{GL}_{2}\left(\mathbb{A}_{f}\right)$ and $r \in \mathrm{GL}_{2}(\widehat{\mathbb{Z}})$. Note that since a Haar measure on the additive group $\operatorname{Mat}_{2}\left(\mathbb{A}_{f}\right)$ is a product-measure satisfying (4.1) with $\beta=2$, we see that $\mu_{2, f}$ is a Haar measure on $\operatorname{Mat}_{2}\left(\mathbb{A}_{f}\right)$.

We denote by $\mu_{\infty}$ the unique $\mathrm{GL}_{2}^{+}(\mathbb{Q})$-invariant measure on $\mathbb{U}$ such that

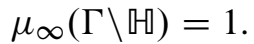

Now put $\mu_{\beta}=2 \mu_{\infty} \times \mu_{\beta, f}$. Then $\mu_{\beta}$ satisfies the conditions in Proposition 3.2, so it corresponds to a $\mathrm{KMS}_{\beta}$-state on the Connes-Marcolli $\mathrm{C}^{*}$-algebra. Indeed, the scaling condition is satisfied since $\prod_{p}|q|_{p}=q^{-1}$ for $q \in \mathbb{Q}_{+}^{*}$. The factor 2 is needed for the normalization condition, since the element $-1 \in \Gamma$ acts trivially on $\mathbb{U}$, while $\mu_{\beta, f}\left(\{ \pm 1\} \backslash \operatorname{Mat}_{2}(\widehat{\mathbb{Z}})\right)=1 / 2$.

Note that the construction of $\mu_{\beta}$ makes sense for all $\beta>1$.

We can now formulate our main result.

Theorem 4.1. For each $\beta \in(1,2]$ the state corresponding to the measure $\mu_{\beta}$ is the unique $K M S_{\beta}$-state on the Connes-Marcolli system.

We shall prove a slightly stronger result which may look more natural if one leaves aside the motivation for the Connes-Marcolli system. Namely, we replace $\mathbb{H}$ by $\mathrm{PGL}_{2}^{+}(\mathbb{R})=\mathrm{GL}_{2}^{+}(\mathbb{R}) / \mathbb{R}^{*}$. Recall that $\mathrm{PGL}_{2}^{+}(\mathbb{R})$ acts transitively on $\mathbb{H}$, and $\mathrm{SO}_{2}(\mathbb{R}) /\{ \pm 1\}$ is the stabilizer of the point $i \in \mathbb{U}$ so that $\mathbb{H}=\mathrm{PGL}_{2}^{+}(\mathbb{R}) / \mathrm{PSO}_{2}(\mathbb{R})$. Denote by $\bar{\mu}_{\infty}$ the Haar measure on $\mathrm{PGL}_{2}^{+}(\mathbb{R})$ normalized such that $\bar{\mu}_{\infty}\left(\Gamma \backslash \mathrm{PGL}_{2}^{+}(\mathbb{R})\right)=1$. Define then a measure on $\operatorname{PGL}_{2}^{+}(\mathbb{R}) \times \operatorname{Mat}_{2}\left(\mathbb{A}_{f}\right)$ by $\bar{\mu}_{\beta}=2 \bar{\mu}_{\infty} \times \mu_{\beta, f}$.

Theorem 4.2. For $\beta \in(1,2]$ the measure $\bar{\mu}_{\beta}$ is the unique $\Gamma$-invariant measure on the space $\mathrm{PGL}_{2}^{+}(\mathbb{R}) \times \operatorname{Mat}_{2}\left(\mathbb{A}_{f}\right)$ such that

$$
\bar{\mu}_{\beta}\left(\Gamma \backslash\left(\mathrm{PGL}_{2}^{+}(\mathbb{R}) \times \operatorname{Mat}_{2}(\widehat{\mathbb{Z}})\right)\right)=1 \quad \text { and } \quad \bar{\mu}_{\beta}(g Z)=\operatorname{det}(g)^{-\beta} \bar{\mu}_{\beta}(Z)
$$

for compact $Z \subset \mathrm{PGL}_{2}^{+}(\mathbb{R}) \times \operatorname{Mat}_{2}\left(\mathbb{A}_{f}\right)$ and $g \in \mathrm{GL}_{2}^{+}(\mathbb{Q})$.

Theorem 4.1 follows from the above theorem since every measure $\mu$ on $\mathbb{H} \times \operatorname{Mat}_{2}\left(\mathbb{A}_{f}\right)$ satisfying the conditions in Proposition 3.2 gives rise to a measure $\bar{\mu}$ on $\mathrm{PGL}_{2}^{+}(\mathbb{R}) \times \operatorname{Mat}_{2}\left(\mathbb{A}_{f}\right)$ satisfying the conditions in Theorem 4.2 by the formula

$$
\int_{\mathrm{PGL}_{2}^{+}(\mathbb{R}) \times \operatorname{Mat}_{2}\left(\mathbb{A}_{f}\right)} f d \bar{\mu}=\int_{\mathbb{W} \times \operatorname{Mat}_{2}\left(\mathbb{A}_{f}\right)}\left(\int_{\mathrm{PSO}_{2}(\mathbb{R})} f(\cdot g) d g\right) d \mu,
$$


where for $x=(h, m) \in \mathrm{PGL}_{2}^{+}(\mathbb{R}) \times \operatorname{Mat}_{2}\left(\mathbb{A}_{f}\right)$ and $g \in \mathrm{PSO}_{2}(\mathbb{R})$ we put $x g=$ $(h g, m)$, and different measures $\mu$ give rise to different $\bar{\mu}$ 's.

Turning to the proof of Theorem 4.2 our first goal is to show uniqueness of $\bar{\mu}_{\beta}$ under the additional assumption of invariance under the right action of $\mathrm{GL}_{2}(\widehat{\mathbb{Z}})$ on $\operatorname{Mat}_{2}\left(\mathbb{A}_{f}\right)$.

Let $F$ be a finite set of prime numbers. Recall that we denote by $S_{F}$ the semigroup of matrices $m \in \operatorname{Mat}_{2}^{+}(\mathbb{Z})$ such that all prime divisors of $\operatorname{det}(m)$ belong to $F$. We then introduce an operator $T_{F}$ on the space of bounded functions on $\Gamma \backslash \mathrm{PGL}_{2}^{+}(\mathbb{R})$ by

$$
\left(T_{F} f\right)(\tau)=\zeta_{S_{F}, \Gamma}(\beta)^{-1} \sum_{s \in \Gamma \backslash S_{F} / \Gamma} \operatorname{det}(s)^{-\beta} R_{\Gamma}(s)\left(T_{s} f\right)(\tau) .
$$

Denote by $\bar{v}_{\infty}$ the measure on $\Gamma \backslash \mathrm{PGL}_{2}^{+}(\mathbb{R})$ defined by $\bar{\mu}_{\infty}$. The following result is a key point in our argument for uniqueness of the $\mathrm{GL}_{2}(\widehat{\mathbb{Z}})$-invariant measure.

Lemma 4.3. For any finite set $J$ of prime numbers, $f \in C_{\mathrm{c}}\left(\Gamma \backslash \mathrm{PGL}_{2}^{+}(\mathbb{R})\right), \varepsilon>0$ and compact subset $\Omega \subset \Gamma \backslash \mathrm{PGL}_{2}^{+}(\mathbb{R})$, there exists a finite set $F$ of prime numbers that is disjoint from $J$ and satisfies

$$
\left|\left(T_{F} f\right)(\tau)-\int_{\Gamma \backslash \mathrm{PGL}_{2}^{+}(\mathbb{R})} f d \bar{v}_{\infty}\right|<\varepsilon \text { for all } \tau \in \Omega .
$$

Proof. By [4, Theorem 1.7] and Remark (3) following it, see also [10] for an alternative proof of a slightly weaker result, there exists a constant $M$ such that

$$
\left|\left(T_{g} f\right)(\tau)-\int_{\Gamma \backslash \mathrm{PGL}_{2}^{+}(\mathbb{R})} f d \bar{v}_{\infty}\right|<\frac{\varepsilon}{2}
$$

for $\tau \in \Omega$ and any $g \in \mathrm{GL}_{2}^{+}(\mathbb{Q})$ with $R_{\Gamma}(g)>M$. We may assume that $M$ is such that $p<M$ for any $p \in J$. Let $F$ be a finite set of prime numbers greater than $M$. Then from Lemma 3.3 we see that $R_{\Gamma}(s)>M$ for any $s \in S_{F}$ such that $\Gamma s \Gamma$ contains a nonscalar diagonal matrix. On the other hand,

$$
\sum_{\substack{s \in \Gamma \backslash S_{F} / \Gamma: \\ s \text { scalar }}} \operatorname{det}(s)^{-\beta}=\prod_{p \in F}\left(\sum_{k=0}^{\infty} p^{-2 \beta k}\right)=\prod_{p \in F}\left(1-p^{-2 \beta}\right)^{-1} \leq \zeta(2 \beta) .
$$

Since the operators $T_{g}$ are contractions in the supremum-norm, we can find $C>0$ such that

$$
\left|\left(T_{g} f\right)(\tau)-\int_{\Gamma \backslash \mathrm{PGL}_{2}^{+}(\mathbb{R})} f d \bar{\nu}_{\infty}\right| \leq C \quad \text { for } \tau \in \Omega \text { and } g \in \mathrm{GL}_{2}^{+}(\mathbb{Q}) .
$$


Therefore by considering separately the summation over double cosets with nonscalar and scalar representatives we get

$$
\left|\left(T_{F} f\right)(\tau)-\int_{\Gamma \backslash \mathrm{PGL}_{2}^{+}(\mathbb{R})} f d \bar{v}_{\infty}\right| \leq \frac{\varepsilon}{2}+\frac{\zeta(2 \beta)}{\zeta_{S_{F}, \Gamma}(\beta)} C \quad \text { for any } \tau \in \Omega .
$$

Recall that by (3.4)

$$
\zeta_{S_{F}, \Gamma}(\beta)=\prod_{p \in F}\left(1-p^{-\beta}\right)^{-1}\left(1-p^{-\beta+1}\right)^{-1}
$$

Since for $\beta \leq 2$ this product diverges as $F$ increases, we see that by choosing sufficiently large $F$ we can make the second summand in the estimate above arbitrarily small, and hence we are done.

We can now analyze the case of measures on $\mathrm{PGL}_{2}^{+}(\mathbb{R}) \times \mathrm{Mat}_{2}\left(\mathbb{A}_{f}\right)$ that are invariant under the right action of $\mathrm{GL}_{2}(\hat{\mathbb{Z}})$ on the second factor.

Lemma 4.4. The measure $\bar{\mu}_{\beta}$ is the unique right $\mathrm{GL}_{2}(\widehat{\mathbb{Z}})$-invariant measure on $\mathrm{PGL}_{2}^{+}(\mathbb{R}) \times \operatorname{Mat}_{2}\left(\mathbb{A}_{f}\right)$ that satisfies the conditions in Theorem 4.2. Furthermore, the action of $\mathrm{GL}_{2}^{+}(\mathbb{Q})$ on the space $\left(\mathrm{PGL}_{2}^{+}(\mathbb{R}) \times\left(\operatorname{Mat}_{2}\left(\mathbb{A}_{f}\right) / \mathrm{GL}_{2}(\widehat{\mathbb{Z}})\right), \bar{\mu}_{\beta}\right)$ is ergodic.

Proof. The measure $\bar{\mu}_{\beta}$ is right $\mathrm{GL}_{2}(\widehat{\mathbb{Z}})$-invariant and satisfies the conditions in Theorem 4.2 by construction. Suppose $\tilde{\mu}$ is another such measure. Let $\tilde{v}$ and $\bar{v}_{\beta}$ be the measures on the quotient space $\Gamma \backslash\left(\mathrm{PGL}_{2}^{+}(\mathbb{R}) \times \operatorname{Mat}_{2}\left(\mathbb{A}_{f}\right)\right)$ defined by $\tilde{\mu}$ and $\bar{\mu}_{\beta}$, respectively. Let $H$ be the subspace of $\operatorname{Mat}_{2}^{+}(\mathbb{Z})$-invariant functions in $L^{2}\left(\Gamma \backslash\left(\mathrm{PGL}_{2}^{+}(\mathbb{R}) \times \operatorname{Mat}_{2}(\hat{\mathbb{Z}})\right), d \tilde{v}\right)$, and denote by $P$ the orthogonal projection onto $H$. Our first goal is to compute how $P$ acts on $\mathrm{GL}_{2}(\widehat{\mathbb{Z}})$-invariant functions.

Let $F$ be a nonempty finite set of prime numbers. Apply Lemma 2.9(2) to the group $G_{F}$, the semigroup $S_{F}$, the set $Y=\operatorname{PGL}_{2}^{+}(\mathbb{R}) \times \operatorname{Mat}_{2}(\widehat{\mathbb{Z}})$ and the subset

$$
Y_{F}=\mathrm{PGL}_{2}^{+}(\mathbb{R}) \times \prod_{p \in F} \mathrm{GL}_{2}\left(\mathbb{Z}_{p}\right) \times \prod_{q \notin F} \operatorname{Mat}_{2}\left(\mathbb{Z}_{q}\right)
$$

in place of $Y_{0}$. Note that we can do this because $S_{F} Y_{F}$ coincides with

$$
\operatorname{PGL}_{2}^{+}(\mathbb{R}) \times \prod_{p \in F} \operatorname{Mat}_{2}^{i}\left(\mathbb{Z}_{p}\right) \times \prod_{q \notin F} \operatorname{Mat}_{2}\left(\mathbb{Z}_{q}\right),
$$

which by Corollary 3.6 (or rather its analogue with $\mathbb{H}$ replaced by $\mathrm{PGL}_{2}^{+}(\mathbb{R})$ ) is a subset of $Y$ of full measure. Thus, denoting by $P_{F}$ the projection onto the subspace of $S_{F}$-invariant functions, for $f_{0} \in L^{2}\left(\Gamma \backslash\left(\mathrm{PGL}_{2}^{+}(\mathbb{R}) \times \mathrm{Mat}_{2}(\widehat{\mathbb{Z}})\right), d \tilde{v}\right)$ we have

$$
\left.P_{F} f_{0}\right|_{S_{F} x}=\zeta_{S_{F}, \Gamma}(\beta)^{-1} \sum_{s \in \Gamma \backslash S_{F} / \Gamma} \operatorname{det}(s)^{-\beta} R_{\Gamma}(s)\left(T_{s} f_{0}\right)(x) \text { for each } x \in Y_{F} .
$$


Given a finite set $J$ of prime numbers which is disjoint from $F$, and a bounded Borel function $f$ on $\Gamma \backslash \mathrm{PGL}_{2}^{+}(\mathbb{R})$, apply (4.3) to the function $f_{0}=f_{J}$, where $f_{J}$ is defined by

$$
f_{J}(x)= \begin{cases}f(\tau) & \text { if } x=(\tau, m) \in Y_{J}, \\ 0 & \text { otherwise. }\end{cases}
$$

Then using the operator $T_{F}$ defined in (4.2), we can write

$$
P_{F} f_{J}=\left(T_{F} f\right)_{J}
$$

Assume now that $f$ is continuous and compactly supported. By Lemma 4.3 we can find a sequence $\left\{F_{n}\right\}_{n}$ of finite sets disjoint from $J$ such that $\left\{T_{F_{n}} f\right\}_{n}$ converges to $\int f d \bar{v}_{\infty}$ uniformly on compact sets. Hence the sequence $\left\{P_{F_{n}} f_{J}\right\}_{n}$ converges weakly in $L^{2}$ to $\int f d \bar{v}_{\infty}\left(\mathbb{1}_{\Gamma \backslash \mathrm{PGL}_{2}^{+}(\mathbb{R})}\right)_{J}=\int f d \bar{v}_{\infty} \mathbb{1}_{\Gamma \backslash Y_{J}}$. Since $P P_{F}=P$ for every $F$, we get

$$
P f_{J}=\int f d \bar{v}_{\infty} P \mathbb{1}_{\Gamma \backslash Y_{J}} .
$$

Using formula (4.3) for the set $J$ instead of $F$, we also see that $P_{J} \mathbb{1}_{\Gamma \backslash Y_{J}}$ is the constant function $\zeta_{S_{J}, \Gamma}(\beta)^{-1}$. Using again that $P P_{J}=P$, we therefore obtain

$$
P f_{J}=\zeta_{S_{J}, \Gamma}(\beta)^{-1} \int_{\Gamma \backslash \mathrm{PGL}_{2}^{+}(\mathbb{R})} f d \bar{\nu}_{\infty} .
$$

Since the space $H$ contains nonzero constant functions, this in particular implies that

$$
\int f_{J} d \tilde{v}=\zeta_{S_{J}, \Gamma}(\beta)^{-1} \int_{\Gamma \backslash \mathrm{PGL}_{2}^{+}(\mathbb{R})} f d \bar{v}_{\infty},
$$

so that $\int f_{J} d \tilde{v}$ is the same for every $\tilde{\mu}$.

To extend the result to all $\mathrm{GL}_{2}(\widehat{\mathbb{Z}})$-invariant functions, fix a finite nonempty set $J$ of prime numbers, and consider a right $\prod_{p \in J} \mathrm{GL}_{2}\left(\mathbb{Z}_{p}\right)$-invariant bounded Borel function $f$ on

$$
\Gamma \backslash\left(\mathrm{PGL}_{2}^{+}(\mathbb{R}) \times \prod_{p \in J} \operatorname{Mat}_{2}\left(\mathbb{Z}_{p}\right)\right) .
$$

We may consider $f$ as a function on $\Gamma \backslash\left(\mathrm{PGL}_{2}^{+}(\mathbb{R}) \times \operatorname{Mat}_{2}(\hat{\mathbb{Z}})\right)$. Then $f$ is right $\mathrm{GL}_{2}(\widehat{\mathbb{Z}})$-invariant, and the space spanned by such functions for all $J$ 's is dense in the space of square integrable $\mathrm{GL}_{2}(\widehat{\mathbb{Z}})$-invariant functions. Applying again formula (4.3) for the projection $P_{J}$ (for $J$ in place of $F$ ), we see that $P_{J} f$ is again a function whose value at $(\tau, m) \in \mathrm{PGL}_{2}^{+}(\mathbb{R}) \times \operatorname{Mat}_{2}(\widehat{\mathbb{Z}})$ depends only on $\tau$ and $m_{p}$ with $p \in J$. The formula also shows that $P_{J}$ commutes with the action of $\mathrm{GL}_{2}(\hat{\mathbb{Z}})$, so $P_{J} f$ is $\mathrm{GL}_{2}(\hat{\mathbb{Z}})$-invariant. Since $\mathrm{GL}_{2}\left(\mathbb{Z}_{p}\right)$ acts transitively on itself, this shows that the value 
of $P_{J} f$ at $(\tau, m)$ with $m_{p} \in \mathrm{GL}_{2}\left(\mathbb{Z}_{p}\right)$ for $p \in J$ depends only on $\tau$. In other words, on the space $\Gamma \backslash Y_{J}$ introduced above, the function $P_{J} f$ is a bounded Borel function of the form $\tilde{f}_{J}$ for some function $\tilde{f}$ on $\Gamma \backslash \mathrm{PGL}_{2}^{+}(\mathbb{R})$. An important point is that $\tilde{f}$ depends on $f$ but not on $\tilde{\mu}$. By Lemma 2.9(1) and the polarization identity we have

$$
\begin{aligned}
\int P_{J} f d \tilde{v} & =\zeta_{S_{J}, \Gamma}(\beta) \int_{\Gamma \backslash Y_{J}} P_{J} f d \tilde{v} \\
& =\zeta_{S_{J}, \Gamma}(\beta) \int_{\Gamma \backslash Y_{J}} \tilde{f}_{J} d \tilde{v} \\
& =\int_{\Gamma \backslash \mathrm{PGL}_{2}^{+}(\mathbb{R})} \tilde{f} d \bar{v}_{\infty} .
\end{aligned}
$$

Since $\int f d \tilde{\nu}=\int P_{J} f d \tilde{\nu}$, we see again that $\int f d \tilde{\nu}$ is the same for any $\tilde{\mu}$. It therefore follows that $\int f d \tilde{v}=\int f d \bar{v}_{\beta}$ for any bounded Borel $\mathrm{GL}_{2}(\widehat{\mathbb{Z}})$-invariant function on $\Gamma \backslash\left(\mathrm{PGL}_{2}^{+}(\mathbb{R}) \times \operatorname{Mat}_{2}(\hat{\mathbb{Z}})\right)$. Since $\tilde{v}$ is $\mathrm{GL}_{2}(\hat{\mathbb{Z}})$-invariant by assumption, we have $\tilde{v}=\bar{v}_{\beta}$ and hence $\tilde{\mu}=\bar{\mu}_{\beta}$.

To prove ergodicity assume that $Z_{0}$ is a left $\mathrm{GL}_{2}^{+}(\mathbb{Q})$-invariant and right $\mathrm{GL}_{2}(\widehat{\mathbb{Z}})$-invariant $\bar{\mu}_{\beta}$-measurable subset of $\mathrm{PGL}_{2}^{+}(\mathbb{R}) \times \mathrm{Mat}_{2}\left(\mathbb{A}_{f}\right)$ of positive measure. Since $\mathrm{GL}_{2}^{+}(\mathbb{Q})\left(\mathrm{PGL}_{2}^{+}(\mathbb{R}) \times \operatorname{Mat}_{2}(\hat{\mathbb{Z}})\right)=\mathrm{PGL}_{2}^{+}(\mathbb{R}) \times \operatorname{Mat}_{2}\left(\mathbb{A}_{f}\right)$, it follows that the set $Z_{0} \cap\left(\mathrm{PGL}_{2}^{+}(\mathbb{R}) \times \operatorname{Mat}_{2}(\widehat{\mathbb{Z}})\right)$ has positive measure. Therefore $\lambda=\bar{\mu}_{\beta}\left(\Gamma \backslash\left(Z_{0} \cap\left(\mathrm{PGL}_{2}^{+}(\mathbb{R}) \times \operatorname{Mat}_{2}(\hat{\mathbb{Z}})\right)\right)\right)>0$. It follows that the measure $\tilde{\mu}$ defined by

$$
\tilde{\mu}(Z)=\lambda^{-1} \bar{\mu}_{\beta}\left(Z_{0} \cap Z\right)
$$

is right $\mathrm{GL}_{2}(\widehat{\mathbb{Z}})$-invariant and satisfies the conditions in Theorem 4.2. Hence $\tilde{\mu}=\bar{\mu}_{\beta}$, and consequently the complement of $Z_{0}$ has $\bar{\mu}_{\beta}$-measure zero.

We aim to prove that the action of $\mathrm{GL}_{2}^{+}(\mathbb{Q})$ on $\left(\mathrm{PGL}_{2}^{+}(\mathbb{R}) \times \operatorname{Mat}_{2}\left(\mathbb{A}_{f}\right), \bar{\mu}_{\beta}\right)$ is ergodic. The next step is to consider the action on $\operatorname{Mat}_{2}\left(\mathbb{A}_{f}\right)$ alone.

Lemma 4.5. The action of $\mathrm{GL}_{2}^{+}(\mathbb{Q})$ on $\left(\operatorname{Mat}_{2}\left(\mathbb{A}_{f}\right), \mu_{\beta, f}\right)$ is ergodic.

Proof. The proof is similar to that of the previous lemma, but requires a much simpler result than Lemma 4.3.

Consider the space $L^{2}\left(\operatorname{Mat}_{2}(\widehat{\mathbb{Z}}), d \mu_{\beta, f}\right)$ and the subspace $H$ of Mat ${ }_{2}^{+}(\mathbb{Z})$-invariant functions. It suffices to show that $H$ consists of constant functions. Denote by $P$ the orthogonal projection onto $H$.

For a finite set $F$ of prime numbers denote by $P_{F}$ the projection onto the space of $S_{F}$-invariant functions. Put also

$$
Y_{F}=\prod_{p \in F} \mathrm{GL}_{2}\left(\mathbb{Z}_{p}\right) \times \prod_{q \notin F} \operatorname{Mat}_{2}\left(\mathbb{Z}_{q}\right)
$$


Then similarly to (4.3) for any $\Gamma$-invariant function $f \in L^{2}\left(\operatorname{Mat}_{2}(\widehat{\mathbb{Z}}), d \mu_{\beta, f}\right)$ we have

$$
\left.P_{F} f\right|_{S_{F} m}=\zeta_{S_{F}, \Gamma}(\beta)^{-1} \sum_{s \in \Gamma \backslash S_{F} / \Gamma} \operatorname{det}(s)^{-\beta} R_{\Gamma}(s)\left(T_{s} f\right)(m) \quad \text { for } m \in Y_{F} .
$$

This can be either proved similarly to Lemma 2.9(2) or deduced from that lemma by identifying the space of $\Gamma$-invariant functions with the subspace of $L^{2}\left(\Gamma \backslash\left(\mathrm{PGL}_{2}^{+}(\mathbb{R}) \times \operatorname{Mat}_{2}(\widehat{\mathbb{Z}})\right), d \bar{v}_{\beta}\right)$ of functions depending only on the second coordinate.

For a finite set $J$ of primes disjoint from $F$, and a left $\Gamma$-invariant function $f$ on $\prod_{p \in J} \mathrm{GL}_{2}\left(\mathbb{Z}_{p}\right)$ define a function $f_{J}$ by

$$
f_{J}(m)= \begin{cases}f\left(\left(m_{p}\right)_{p \in J}\right) & \text { if } m_{p} \in \mathrm{GL}_{2}\left(\mathbb{Z}_{p}\right) \text { for } p \in J \\ 0 & \text { otherwise. }\end{cases}
$$

Since $f$ is $\Gamma$-invariant and $\Gamma$ is dense in $\prod_{p \in J} \operatorname{SL}_{2}\left(\mathbb{Z}_{p}\right), f$ is invariant with respect to multiplication on the left by elements of the latter group. In other words, the value of $f$ at $m$ depends only on $\operatorname{det}(m) \in \prod_{p \in J} \mathbb{Z}_{p}^{*}$. Therefore functions of the form $f(m)=\chi(\operatorname{det}(m))$, where $\chi$ is a character of the compact abelian group $\prod_{p \in J} \mathbb{Z}_{p}^{*}$, span a dense subspace of $\Gamma$-invariant functions on $\prod_{p \in J} \mathrm{GL}_{2}\left(\mathbb{Z}_{p}\right)$. But if $f=\chi \circ$ det, we have

$$
\left(T_{s} f\right)(m)=\chi(\operatorname{det}(m)) \chi(\operatorname{det}(s))
$$

for $s \in S_{F}$ and $m \in \prod_{p \in J} \mathrm{GL}_{2}\left(\mathbb{Z}_{p}\right)$. Applying now (4.5) to the function $f_{J}$ and using a calculation similar to (3.2) and (3.4), we get

$$
\begin{aligned}
\left.P_{F} f_{J}\right|_{S_{F} m} & =\chi\left(\operatorname{det}\left(\left(m_{p}\right)_{p \in J}\right)\right) \zeta_{S_{F}, \Gamma}(\beta)^{-1} \sum_{s \in \Gamma \backslash S_{F} / \Gamma} \operatorname{det}(s)^{-\beta} R_{\Gamma}(s) \chi(\operatorname{det}(s)) \\
& =\chi\left(\operatorname{det}\left(\left(m_{p}\right)_{p \in J}\right)\right) \prod_{p \in F} \frac{\left(1-p^{-\beta}\right)\left(1-p^{-\beta+1}\right)}{\left(1-\chi(p) p^{-\beta}\right)\left(1-\chi(p) p^{-\beta+1}\right)} .
\end{aligned}
$$

If the character $\chi$ is nontrivial, by choosing $F$ large enough the product above can be made arbitrarily small by elementary properties of Dirichlet series (this was used already for the classification of KMS-states of the Bost-Connes system in [3], see also [18]). Since $P P_{F}=P$, we conclude that $P f_{J}=0$. On the other hand, if $\chi$ is trivial then $f_{J}=\mathbb{1}_{Y_{J}}$. Then applying (4.5) with $J$ in place of $F$ we get $P_{J} f_{J}=\zeta_{S_{J}, \Gamma}(\beta)^{-1}$. In either case we see that $P f_{J}$ is constant.

Let now $f$ be a function on $\prod_{p \in J} \mathrm{GL}_{2}\left(\mathbb{Z}_{p}\right)$ which is no longer left $\Gamma$-invariant. Since $\Gamma$ is dense in $\mathrm{SL}_{2}(\widehat{\mathbb{Z}})$, any function in $H$ is $\mathrm{SL}_{2}(\widehat{\mathbb{Z}})$-invariant. Hence to compute $P f_{J}$ we can first apply to $f_{J}$ the projection $Q$ onto the subspace of $\mathrm{SL}_{2}(\hat{\mathbb{Z}})$-invariant 
functions. But $Q$ is given by averaging over $\mathrm{SL}_{2}(\hat{\mathbb{Z}})$-orbits. We then observe that $Q f_{J}=\tilde{f}_{J}$, where

$$
\tilde{f}(m)=\int_{\prod_{p \in J} \mathrm{SL}_{2}\left(\mathbb{Z}_{p}\right)} f(g m) d g .
$$

Hence $P f_{J}=P Q f_{J}=P \tilde{f}_{J}$ is again a constant function.

To extend the result to all functions on $\operatorname{Mat}_{2}(\widehat{\mathbb{Z}})$, for each $s \in \operatorname{Mat}_{2}^{+}(\mathbb{Z})$ we introduce an operator $V_{s}$ on the space $L^{2}\left(\operatorname{Mat}_{2}(\widehat{\mathbb{Z}}), d \mu_{\beta, f}\right)$ by letting $\left(V_{s} h\right)(m)=$ $h(\mathrm{sm})$. Then $V_{s} P=P$. Using the scaling condition we see that $\operatorname{det}(s)^{-\beta / 2} V_{s}$ is a coisometry with initial space $L^{2}\left(s \operatorname{Mat}_{2}(\widehat{\mathbb{Z}}), d \mu_{\beta, f}\right)$. It follows that the adjoint operator is given by

$$
\left(V_{s}^{*} h\right)(y)= \begin{cases}\operatorname{det}(s)^{\beta} h\left(s^{-1} y\right) & \text { if } y \in s \operatorname{Mat}_{2}(\widehat{\mathbb{Z}}) \\ 0 & \text { otherwise }\end{cases}
$$

In particular, we see that if $s \in S_{J}$ for some finite set $J$ then both operators $V_{s}$ and $V_{s}^{*}$ preserve the space of functions $f$ such that $f(m)$ depends only on $m_{p}$ with $p \in J$. But then if $f$ is such a function with support on $Y_{J}$, the function $V_{s}^{*} f$ has support on $s Y_{J}$. Since $V_{s} P=P$, we have $P V_{s}^{*}=P$ and thus $P V_{s}^{*} f=P f$ is a constant. We thus see that the image of a dense space of functions consists of constant functions.

The following simple trick will allow us to combine the two previous lemmas. It expounds a remark in [18].

Proposition 4.6. Assume we have mutually commuting actions of locally compact second countable groups $G_{1}, G_{2}$ and $G_{3}$ on a Lebesgue space $(X, \mu)$. Suppose that

(i) the actions of $G_{1}$ on $\left(X / G_{2}, \mu\right)$ and $\left(X / G_{3}, \mu\right)$ are ergodic;

(ii) $G_{2}$ is connected and $G_{3}$ is compact totally disconnected.

Then the action of $G_{1}$ on $(X, \mu)$ is ergodic.

Here by quotient spaces we mean quotients in measure theoretic sense. So by definition

$$
L^{\infty}\left(X / G_{i}, \mu\right)=L^{\infty}(X, \mu)^{G_{i}} .
$$

Proof of Proposition 4.6. By assumption the action of $G_{1} \times G_{3}$ on $X$ is ergodic. In other words, the action of $G_{3}$ on $X / G_{1}$ is ergodic. Since $G_{3}$ is compact, we can then identify $X / G_{1}$ with a homogeneous space of $G_{3}$, say $G_{3} / H$, where $H$ is a closed subgroup of $G_{3}$, see e.g. [23], Section 2.1. Since $G_{1} \times G_{2}$ acts ergodically on $X$, we have an ergodic action of $G_{2}$ on $X / G_{1}=G_{3} / H$. Since this action commutes with the action of $G_{3}$ on $G_{3} / H$ by left translations, it is given by right translations, that is, by a measurable homomorphism $G_{2} \rightarrow N(H) / H$, where $N(H)$ 
is the normalizer of $H$ in $G_{3}$. Such a homomorphism is automatically continuous (see e.g. [23], Theorem B.3), and since $G_{2}$ is connected and $N(H) / H$ is totally disconnected, the homomorphism must be trivial. But since the action of $G_{2}$ is ergodic this means that $H=G_{3}$, so that $X / G_{1}$ is a single point. Thus the action of $G_{1}$ is ergodic.

Corollary 4.7. The left action of $\mathrm{GL}_{2}^{+}(\mathbb{Q})$ on $\left(\mathrm{PGL}_{2}^{+}(\mathbb{R}) \times \operatorname{Mat}_{2}\left(\mathbb{A}_{f}\right), \bar{\mu}_{\beta}\right)$ is ergodic.

Proof. The proof is a straightforward application of Proposition 4.6 with $G_{1}=\mathrm{GL}_{2}^{+}(\mathbb{Q}), G_{2}=\mathrm{PGL}_{2}^{+}(\mathbb{R})$ and $G_{3}=\mathrm{GL}_{2}(\hat{\mathbb{Z}})$, so that $G_{1}$ acts on $\mathrm{PGL}_{2}^{+}(\mathbb{R}) \times \operatorname{Mat}_{2}\left(\mathbb{A}_{f}\right)$ by multiplication on the left and $G_{2}$ and $G_{3}$ act by multiplication on the right on the corresponding factor. That the actions of $G_{1}$ on the quotient spaces are ergodic is given by Lemma 4.4 and Lemma 4.5.

Proof of Theorem 4.2. We follow an argument similar to that of [3], Theorem 25. Note first that $\bar{\mu}_{\beta}$ is right $\mathrm{GL}_{2}(\hat{\mathbb{Z}})$-invariant and satisfies the conditions in Theorem 4.2 by construction. Denote by $K_{\beta}$ the affine set of measures on $\mathrm{PGL}_{2}^{+}(\mathbb{R}) \times \operatorname{Mat}_{2}\left(\mathbb{A}_{f}\right)$ satisfying the conditions in Theorem 4.2. Let $C_{\beta}$ be a cone with base $K_{\beta}$. Denote by $v_{0}$ its vertex. The cone $C_{\beta}$ has the structure of a Choquet simplex. Namely, similarly to Proposition 3.2 it can be identified with the set of $\mathrm{KMS}_{\beta}$-states on $B^{\sim}$, where

$$
B=C_{\mathrm{r}}^{*}\left(\Gamma \backslash \mathrm{GL}_{2}^{+}(\mathbb{Q}) \bigotimes_{\Gamma}\left(\mathrm{PGL}_{2}^{+}(\mathbb{R}) \times \operatorname{Mat}_{2}(\hat{\mathbb{Z}})\right)\right),
$$

$B^{\sim}$ is obtained from $B$ by adjoining a unit, and $v_{0}$ corresponds to the state on $B^{\sim}$ with kernel $B$. Denote by $\bar{\varphi}$ the state corresponding to $\bar{\mu}_{\beta}$. Then by Remark 2.3 the algebra $\pi_{\bar{\varphi}}\left(B^{\sim}\right)^{\prime \prime}$ is a reduction of the von Neumann algebra of the orbit equivalence relation defined by the action of $\mathrm{GL}_{2}^{+}(\mathbb{Q})$ on $\left(\mathrm{PGL}_{2}^{+}(\mathbb{R}) \times \operatorname{Mat}_{2}\left(\mathbb{A}_{f}\right), \bar{\mu}_{\beta}\right)$. By Corollary 4.7 this von Neumann algebra is a factor. Hence $\pi_{\bar{\varphi}}\left(B^{\sim}\right)^{\prime \prime}$ is also a factor, and therefore $\bar{\mu}_{\beta}$ is an extremal point of $C_{\beta}$. The group $\mathrm{GL}_{2}(\widehat{\mathbb{Z}})$ acts on $C_{\beta}$, and by virtue of Lemma 4.4 the segment $\left[\bar{\mu}_{\beta}, v_{0}\right]$ is the set of $\mathrm{GL}_{2}(\hat{\mathbb{Z}})$-invariant points. Suppose now $v \in C_{\beta}$ is an extremal point. Then $w=\int_{\mathrm{GL}_{2}(\hat{\mathbb{Z}})} g v d g \in C_{\beta}$ where each $g v$ is also an extremal point of $C_{\beta}$. But because of its $\mathrm{GL}_{2}(\hat{\mathbb{Z}})$ invariance, $w$ lies on $\left[\bar{\mu}_{\beta}, v_{0}\right]$ and hence is also a convex combination of the extremal points $\bar{\mu}_{\beta}$ and $v_{0}$. Since $w$ is the barycenter of a unique probability measure on the set of extremal points, we conclude that either $v=\bar{\mu}_{\beta}$ or $v=v_{0}$. Thus $C_{\beta}=\left[\bar{\mu}_{\beta}, v_{0}\right]$ and $K_{\beta}=\left\{\bar{\mu}_{\beta}\right\}$. This completes the proof of Theorem 4.2.

Remark 4.8. We have classified $\mathrm{KMS}_{\beta}$-states of the Connes-Marcolli system for $\beta \neq 0,1$. Let us now briefly discuss the cases $\beta=0,1$.

(i) If $\beta=0$ then by Lemma 3.5 and the considerations following Corollary 3.6 one can conclude that there are no nonzero finite traces on $I=C_{\mathrm{r}}^{*}\left(\Gamma \backslash \mathrm{GL}_{2}^{+}(\mathbb{Q}) \bigotimes_{\Gamma}\right.$ $\left.\left(\mathbb{H} \times \operatorname{Mat}_{2}(\widehat{\mathbb{Z}})^{\times}\right)\right)$. Therefore the only $\mathrm{KMS}_{0}$-states, that is, $\sigma$-invariant traces, 
are those coming from $A / I=C_{\mathrm{r}}^{*}\left(\Gamma \backslash \mathrm{GL}_{2}^{+}(\mathbb{Q}) \times_{\Gamma} \mathbb{H}\right)$. There is a canonical trace defined by the $\mathrm{GL}_{2}^{+}(\mathbb{Q})$-invariant measure $\mu_{\infty}$ on $\mathbb{H}$. Notice that though the action of $\mathrm{GL}_{2}^{+}(\mathbb{Q})$ on $\mathbb{H}$ is not free and so Proposition 2.1 is not immediately applicable, the action of $\mathrm{GL}_{2}^{+}(\mathbb{Q}) / \mathbb{Q}^{*}$ is free in the measure theoretic sense, and this is enough to check the trace property. This is probably the unique such trace.

(ii) If $\beta=1$ then, as we know, $\mathrm{KMS}_{1}$-states still correspond to measures satisfying the scaling condition. By the first part of the proof of Corollary 3.6 and our considerations following that corollary, the set of points $(\tau, m) \in \mathbb{U} \times \operatorname{Mat}_{2}\left(\mathbb{A}_{f}\right)$ with $m_{p} \neq 0, \operatorname{det}\left(m_{p}\right)=0$ for every $p$, is a subset of full measure. Such measures indeed exist. Let $\mu_{f}^{\prime}$ be the Haar measure on the locally compact group $\mathbb{A}_{f}^{2}$ normalized such that $\mu_{f}^{\prime}\left(\hat{\mathbb{Z}}^{2}\right)=1$. We may consider $\mu_{f}^{\prime}$ as a measure on $\operatorname{Mat}_{2}\left(\mathbb{A}_{f}\right)$ by identifying $\mathbb{A}_{f}^{2}$ with the set of matrices with zero first column. Then $\mu^{\prime}=2 \mu_{\infty} \times \mu_{f}^{\prime}$ is a measure with the required properties. Using the action of $\mathrm{GL}_{2}(\hat{\mathbb{Z}})$ by multiplication on the right we can then construct infinitely many such measures (notice that the stabilizer of $\mu^{\prime}$ in $\mathrm{GL}_{2}(\widehat{\mathbb{Z}})$ is the group of upper triangular matrices). We conjecture that this way one gets all extremal $\mathrm{KMS}_{1}$-states.

Remark 4.9. Let $1<\beta \leq 2$, and denote by $\varphi_{\beta}$ the unique $\mathrm{KMS}_{\beta}$-state on the Connes-Marcolli $\mathrm{C}^{*}$-algebra $A$. It is easy to describe the flow of weights of the factor $\pi_{\varphi_{\beta}}(A)^{\prime \prime}$. Let us first consider $B=C_{\mathrm{r}}^{*}\left(\Gamma \backslash \mathrm{GL}_{2}^{+}(\mathbb{Q}) \bigotimes_{\Gamma}\left(\mathrm{PGL}_{2}^{+}(\mathbb{R}) \times \operatorname{Mat}_{2}(\hat{\mathbb{Z}})\right)\right)$ and the state $\bar{\varphi}_{\beta}$ on $B$ corresponding to $\bar{\mu}_{\beta}$, and describe the flow of weights of $\pi_{\bar{\varphi}_{\beta}}(B)^{\prime \prime}$. By Remark 2.3, equivalently we want to describe the flow of weights of the orbit equivalence relation defined by the ergodic action of $\mathrm{GL}_{2}^{+}(\mathbb{Q})$ on $\left(\mathrm{PGL}_{2}^{+}(\mathbb{R}) \times \operatorname{Mat}_{2}\left(\mathbb{A}_{f}\right), \bar{\mu}_{\beta}\right)$.

$\mathbb{R}_{+}^{*}$ acts on the measure space $\left(\mathbb{R}_{+}^{*} \times \operatorname{PGL}_{2}^{+}(\mathbb{R}) \times \operatorname{Mat}_{2}\left(\mathbb{A}_{f}\right), \lambda \times \bar{\mu}_{\infty} \times \mu_{\beta, f}\right)$, where $\lambda$ is a measure in the Lebesgue measure class, by

$$
t(s, h, \rho)=\left(t^{-1 / \beta} s, h, \rho\right) .
$$

The flow of weights is induced by this action on the quotient of the space by the action of $\mathrm{GL}_{2}^{+}(\mathbb{Q})$ defined by

$$
g(s, h, \rho)=(\operatorname{det}(g) s, g h, g \rho)
$$

We have an isomorphism $\mathrm{GL}_{2}^{+}(\mathbb{R}) /\{ \pm 1\} \rightarrow \mathbb{R}_{+}^{*} \times \mathrm{PGL}_{2}^{+}(\mathbb{R}), g \mapsto(\operatorname{det}(g), \bar{g})$, where $\bar{g}$ denotes the class of $g$ in $\mathrm{PGL}_{2}^{+}(\mathbb{R})$. So instead of the space $\mathbb{R}_{+}^{*} \times \mathrm{PGL}_{2}^{+}(\mathbb{R}) \times$ $\operatorname{Mat}_{2}\left(\mathbb{A}_{f}\right)$ we may consider $\left(G_{2}^{+}(\mathbb{R}) /\{ \pm 1\}\right) \times \operatorname{Mat}_{2}\left(\mathbb{A}_{f}\right)$. We may further replace $\mathrm{GL}_{2}^{+}(\mathbb{R})$ by $\mathrm{GL}_{2}(\mathbb{R})$, but instead of the action of $\mathrm{GL}_{2}^{+}(\mathbb{Q})$ we then have to consider the action of $\mathrm{GL}_{2}(\mathbb{Q})$. Finally, replace $\mathrm{GL}_{2}(\mathbb{R})$ by $\operatorname{Mat}_{2}(\mathbb{R})$, and so instead of 
$\mathrm{GL}_{2}(\mathbb{R}) \times \operatorname{Mat}_{2}\left(\mathbb{A}_{f}\right)$ consider $\operatorname{Mat}_{2}(\mathbb{A})$, where $\mathbb{A}=\mathbb{R} \times \mathbb{A}_{f}$ is the full adele space. To summarize, $\mathbb{R}_{+}^{*}$ acts on $\operatorname{Mat}_{2}(\mathbb{A})=\operatorname{Mat}_{2}(\mathbb{R}) \times \operatorname{Mat}_{2}\left(\mathbb{A}_{f}\right)$ by $t(m, \rho)=\left(t^{-1 / 2 \beta} m, \rho\right)$, and the flow of weights of the factor $\pi_{\bar{\varphi}_{\beta}}(B)^{\prime \prime}$ is induced by this action on the quotient of the measure space $\left(\operatorname{Mat}_{2}(\mathbb{R}) \times \operatorname{Mat}_{2}\left(\mathbb{A}_{f}\right), \lambda_{\infty} \times \mu_{\beta, f}\right)$, where $\lambda_{\infty}$ is the usual Lebesgue measure on $\operatorname{Mat}_{2}(\mathbb{R}) \cong \mathbb{R}^{4}$, by the action of $\mathrm{GL}_{2}(\mathbb{Q}) \times\{ \pm 1\}$ defined by $(g, s)(m, \rho)=(g m s, g \rho)$.

Denote the measure $\lambda_{\infty} \times \mu_{\beta, f}$ on $\operatorname{Mat}_{2}(\mathbb{A})$ by $\lambda_{\beta}$. Note that $\lambda_{2}$ is a Haar measure on the additive group $\operatorname{Mat}_{2}(\mathbb{A})$.

Similarly, by identifying $\mathbb{R}_{+}^{*} \times \mathbb{W}$ with $\mathrm{GL}_{2}^{+}(\mathbb{R}) / \mathrm{SO}_{2}(\mathbb{R})$ we conclude that the flow of weights of the factor $\pi_{\varphi_{\beta}}(A)^{\prime \prime}$ is defined on the quotient of the measure space $\left(\operatorname{Mat}_{2}(\mathbb{A}), \lambda_{\beta}\right)$ by the action of $\mathrm{GL}_{2}(\mathbb{Q}) \times \mathrm{SO}_{2}(\mathbb{R})$ defined by $(g, s)(m, \rho)=(g m s, g \rho)$ for $(g, s) \in \mathrm{GL}_{2}(\mathbb{Q}) \times \mathrm{SO}_{2}(\mathbb{R})$ and $(m, \rho) \in \operatorname{Mat}_{2}(\mathbb{A})=$ $\operatorname{Mat}_{2}(\mathbb{R}) \times \operatorname{Mat}_{2}\left(\mathbb{A}_{f}\right)$.

It seems natural to conjecture that the action of $\mathrm{GL}_{2}(\mathbb{Q})$ on $\left(\operatorname{Mat}_{2}(\mathbb{A}), \lambda_{\beta}\right)$ is ergodic, so the flows of weights of the factors $\pi_{\varphi_{\beta}}(A)^{\prime \prime}$ and $\pi_{\bar{\varphi}_{\beta}}(B)^{\prime \prime}$ are trivial, and thus the factors are of type $\mathrm{III}_{1}$. The analogous property in the one-dimensional case indeed holds [3], [18]. Note that so far we have only shown that the action of $\mathrm{GL}_{2}(\mathbb{Q}) \times \mathbb{R}^{*}$ is ergodic, which is equivalent to ergodicity of the action of $\mathrm{GL}_{2}^{+}(\mathbb{Q})$ on $\left(\mathrm{PGL}_{2}^{+}(\mathbb{R}) \times \operatorname{Mat}_{2}\left(\mathbb{A}_{f}\right), \bar{\mu}_{\beta}\right)$. Note also that similarly to the one-dimensional case [18], by virtue of Lemma 4.5 and Proposition 4.6, to prove the conjecture it would be enough to show that the action of $\mathrm{GL}_{2}^{+}(\mathbb{Q})$ on $\mathrm{GL}_{2}^{+}(\mathbb{R}) \times\left(\operatorname{Mat}_{2}\left(\mathbb{A}_{f}\right) / \mathrm{GL}_{2}(\hat{\mathbb{Z}})\right)$ is ergodic, or equivalently, the action of $\mathrm{GL}_{2}(\mathbb{Q})$ on $\left(\operatorname{Mat}_{2}(\mathbb{A}) / \mathrm{GL}_{2}(\widehat{\mathbb{Z}}), \lambda_{\beta}\right)$ is ergodic. Recall that in the one-dimensional case the corresponding ergodicity result for the action of $\mathbb{Q}^{*}$ on $\mathbb{A} / \widehat{\mathbb{Z}}^{*}$ was established in [1] and [2].

Remark 4.10. We believe that the results of Sections 3 and 4 are valid for $\mathrm{GL}_{n}$ for any $n \geq 2$. Consider the algebra $C_{\mathrm{r}}^{*}\left(\mathrm{SL}_{n}(\mathbb{Z}) \backslash \mathrm{GL}_{n}^{+}(\mathbb{Q}) \bigotimes_{\mathrm{SL}_{n}(\mathbb{Z})}\left(\mathrm{PGL}_{n}^{+}(\mathbb{R}) \times \operatorname{Mat}_{n}(\hat{\mathbb{Z}})\right)\right)$. Define a dynamics by the homomorphism $\mathrm{GL}_{n}^{+}(\mathbb{Q}) \ni g \mapsto \operatorname{det}(g)$. Then

(i) for $\beta \in(-\infty, 0) \cup(0,1) \cup \cdots \cup(n-2, n-1)$ there are no $\mathrm{KMS}_{\beta}$-states;

(ii) for $\beta \in(n-1, n]$ there exists a unique $\mathrm{KMS}_{\beta}$-state;

(iii) for $\beta>n$ there is a one-to-one correspondence between $\mathrm{KMS}_{\beta}$-states and probability measures on $\mathrm{SL}_{n}(\mathbb{Z}) \backslash\left(\mathrm{PGL}_{n}^{+}(\mathbb{R}) \times \mathrm{GL}_{n}(\hat{\mathbb{Z}})\right)$;

(iv) for $\beta=0,1, \ldots, n-1$ there is a $\mathrm{KMS}_{\beta}$-state defined by the Haar measure on $\mathbb{A}_{f}^{\beta n}$, when we identify the latter group with the set of matrices in $\operatorname{Mat}_{n}\left(\mathbb{A}_{f}\right)$ with zero first $n-\beta$ columns.

The key step for this generalization would be an analogue of Lemma 3.5. 


\section{References}

[1] B. Blackadar, The regular representation of restricted direct product groups. J. Funct. Analysis 25 (1977), 267-274. Zbl 0364.22004 MR 0439979

[2] F. P. Boca and A. Zaharescu, Factors of type III and the distribution of prime numbers. Proc. London Math. Soc. (3) 80 (2000), 145-178. Zbl 1029.46096 MR 1719164

[3] J.-B. Bost and A. Connes, Hecke algebras, type III factors and phase transitions with spontaneous symmetry breaking in number theory. Selecta Math. (N.S.) 1 (1995), 411-457. Zbl 0842.46040 MR 1366621

[4] L. Clozel, H. Oh, and E. Ullmo, Hecke operators and equidistribution of Hecke points. Invent. Math. 144 (2001), 327-351. Zbl 01655627 MR 1827734

[5] A. Connes and M. Marcolli, Quantum statistical mechanics of $\mathbb{Q}$-lattices (from physics to number theory via noncommutative geometry, part I). In Frontiers in Number Theory, Physics, and Geometry I (P. Cartier, B. Julia, P. Moussa, P. Vanhove, eds.), second printing, Springer-Verlag, Berlin 2006, 269-349. MR 2261099

[6] A. Connes and M. Marcolli, Q-lattices: quantum statistical mechanics and Galois theory. J. Geom. Phys. 56 (2006), 2-23. Zbl 02235668 MR 2170598

[7] A. Connes, M. Marcolli, and N. Ramachandran, KMS states and complex multiplication. Selecta Math. (N.S.) 11 (2005), 325-347. Zbl 1106.58005 MR 2215258

[8] A. Connes, M. Marcolli, and N. Ramachandran, KMS states and complex multiplication. II. In Operator Algebras: The Abel Symposium 2004, Abel Symp. 1, Springer-Verlag, Berlin 2006, 15-59. Zbl 05130838 MR 2265042

[9] A. Connes and H. Moscovici, Modular Hecke algebras and their Hopf symmetry. Mosc. Math. J. 4 (2004), 67-109. Zbl 02142711 MR 2074984

[10] A. Eskin and H. Oh, Ergodic theoretic proof of equidistribution of Hecke points. Ergodic Theory Dynam. Systems 26 (2006), 163-167. Zbl 1092.11023 MR 2201942

[11] J. Feldman and C. C. Moore, Ergodic equivalence relations, cohomology, and von Neumann algebras. I, II. Trans. Amer. Math. Soc. 234 (1977), 289-324; ibid. 325-359. Zbl 0369.22009 Zbl 0369.22010; MR 0578656 MR 0578730

[12] E. Ha and F. Paugam, Bost-Connes-Marcolli systems for Shimura varieties. Part I. Definitions and formal analytic properties. Internat. Math. Res. Papers 2005 (2005), 237-286. Zbl 05034362 MR 2199962

[13] A. Krieg, Hecke algebras. Mem. Amer. Math. Soc. 87 (1990), no. 435. Zbl 0706.11029 MR 1027069

[14] J. Kustermans, KMS-weights on $C^{*}$-algebras. Preprint 1997. arXiv:funct-an/9704008

[15] M. Laca, Semigroups of *-endomorphisms, Dirichlet series, and phase transitions. J. Funct. Anal. 152 (1998), 330-378. Zbl 0957.46039 MR 1608003

[16] M. Laca and S. Neshveyev, KMS states of quasi-free dynamics on Pimsner algebras. $J$. Funct. Anal. 211 (2004), 457-482. Zbl 1060.46049 MR 2056837

[17] P. S. Muhly, J. N. Renault and D. P. Williams, Equivalence and isomorphism for groupoid $C^{*}$-algebras. J. Operator Theory 17 (1987), 3-22. Zbl 0645.46040 MR 873460 
[18] S. Neshveyev, Ergodicity of the action of the positive rationals on the group of finite adeles and the Bost-Connes phase transition theorem. Proc. Amer. Math. Soc. 130 (2002), 2999-3003. Zbl 1031.46077 MR 1908923

[19] J. Renault, A groupoid approach to $C^{*}$-algebras. Lecture Notes in Math. 793, SpringerVerlag, Berlin 1980. Zbl 0433.46049 MR 0584266

[20] G. Shimura, Introduction to the arithmetic theory of automorphic functions. Publ. Math. Soc. Japan 11, Iwanami Shoten Publishers, Tokyo; Princeton University Press, Princeton, N.J., 1971. Zbl 0221.10029 MR 0314766

[21] K. Tzanev, Cross product by Hecke pairs. Talk at "Workshop on Noncommutative Geometry and Number Theory, II”, Max-Planck-Institut für Mathematik, Bonn, June 14-18, 2004.

[22] A. Weil, Basic number theory. 3rd ed., Grundlehren Math. Wiss. 144, Springer-Verlag, Berlin 1974. Zbl 0326.12001 MR 0427267

[23] R. J. Zimmer, Ergodic theory and semisimple groups. Monogr. Math. 81, Birkhäuser, Basel 1984. Zbl 0571.58015 MR 0776417

Received September 11, 2006

M. Laca, Department of Mathematics and Statistics, University of Victoria, P.O. Box 3045, Victoria, British Columbia, V8W 3P4, Canada

E-mail: laca@math.uvic.ca

N. S. Larsen, S. Neshveyev, Department of Mathematics, University of Oslo, P.O. Box 1053, Blindern, 0316 Oslo, Norway

E-mail: nadias1@math.uio.no; sergeyn@math.uio.no 